ApJ IN PREss - REVISED AFter REFEREE's ChANGES

Preprint typeset using IATEX style emulateapj v. 25/04/01

\title{
A COMPARISON OF THE GALAXY POPULATIONS IN THE COMA AND DISTANT CLUSTERS: THE EVOLUTION OF K+A GALAXIES AND THE ROLE OF THE INTRACLUSTER MEDIUM
}

\author{
Bianca M. Poggianti ${ }^{2}$ Terry J. Bridges, ${ }^{3}$ Y. Komiyama,${ }^{4}$ M. Yagi,${ }^{5}$ Dave Carter,${ }^{6}$ \\ Bahram Mobasher, ${ }^{7}$ S. OKamura, ${ }^{4}$ N. Kashikawa ${ }^{5}$ \\ 2) Osservatorio Astronomico di Padova, vicolo dell'Osservatorio 5, 35122 Padova, Italy, poggianti@pd.astro.it \\ 3) Anglo-Australian Observatory, PO Box 296, Epping, NSW 2121, Australia \\ 4) Department of Astronomy, University of Tokyo, Bunkyo-ku, Tokyo 113-0033, Japan \\ 5) National Astronomical Observatory, Mitaka, Tokyo, 181-8588 Japan \\ 6) Liverpool John Moores University, Astrophysics Research Institute, Twelve Quays House, Egerton Wharf, \\ Birkenhead, Wirral, CH41 1LD, UK \\ 7) Space Telescope Science Institute, 3700 San Martin Drive, Baltimore MD 21218, USA \\ Affiliated with the Space Sciences Department of the European Space Agency \\ ApJ in press - Revised after referee's changes
}

\begin{abstract}
The spectroscopic properties of galaxies in the Coma cluster are compared with those of galaxies in rich clusters at $z \sim 0.5$, to investigate the evolution of the star formation history in clusters. Luminous galaxies with $M_{V} \leq-20$ and post-starburst/post-starforming $(\mathrm{k}+\mathrm{a})$ spectra which constitute a significant fraction of galaxies in distant cluster samples are absent in Coma, where spectacular cases of $\mathrm{k}+\mathrm{a}$ spectra are found instead at $M_{V}>-18.5$ and represent a significant proportion of the cluster dwarf galaxy population. A simple inspection of their positions on the sky indicates that this type of galaxy does not show a preferential location within the cluster, but the bluest and strongest-lined group of $\mathrm{k}+\mathrm{a}$ 's lies in projection towards the central $1.4 \mathrm{Mpc}$ of Coma and have radial velocities significantly higher than the cluster mean. We find a striking correlation between the positions of these young and strong post-starburst galaxies and substructure in the hot intracluster medium (ICM) identified from $X M M-N e w t o n$ data, with these galaxies lying close to the edges of two infalling substructures. This result strongly suggests that the interaction with the dense ICM could be responsible for the quenching of the star formation (thus creating the $\mathrm{k}+\mathrm{a}$ spectrum), and possibly, for any previous starburst. The evolution with redshift of the luminosity distribution of $\mathrm{k}+\mathrm{a}$ galaxies can be explained by a "downsizing effect", with the maximum luminosity/mass of actively star-forming galaxies infalling onto clusters decreasing at lower redshift. We discuss the possible physical origin of this downsizing effect and the implications of our results for current scenarios of environmental effects on the star formation in galaxies.
\end{abstract}

Subject headings: galaxies: clusters-galaxies: clusters: individual(Coma) — galaxies: evolution

\section{INTRODUCTION}

Fundamental to our understanding of how and why galaxies evolve is a detailed knowledge of how their stellar content evolves and what physical processes drive these changes. Over the years, solid observational evidence has accumulated indicating that processes external to galaxies can be as important as, and may be in some cases more important than, intrinsic processes operating inside galaxies. The average stellar content of galaxies changes profoundly as a function of environment, attesting that the build-up of stellar populations occurred in a fashion that was greatly influenced by environmental conditions. In order to study galaxy evolution, clusters of galaxies are therefore excellent places to start with, because their virialized and infall regions allow us to probe a large range of physical conditions and can be used to test the relative roles of intrinsic evolution, of the local environment around a galaxy, and of the global environment, on a group-, filament- and clusterwide scale, over a wide range of structure masses.

The evolution of galaxies in clusters has been investigated through observations of distant clusters using galaxy colors (Butcher \& Oemler 1984; Ellingson et al. 2001; Ko- dama \& Bower 2001; Stanford et al. 1998; Kodama et al. 1998), morphologies (Dressler et al. 1997; Fasano et al. 2000; van Dokkum et al. 2000; Lubin et al. 2002), scaling relations (Kelson et al. 1997, 2000; Ziegler \& Bender 1997) and spectral properties (Couch and Sharples 1987; Dressler \& Gunn 1992; Abraham et al. 1996; Fisher et al. 1998; Dressler et al. 1999; Balogh et al. 1999; Ellingson et al. 2001; Postman et al. 2001). In particular, galaxy spectra carry a wealth of information about the current and past star formation activity in galaxies and, in this regard, are superior to photometry for studying the evolutionary histories of galaxies.

Spectroscopic surveys of galaxies in distant clusters have detected high fractions of currently or recently starforming galaxies at high-z (Couch and Sharples 1987; Fisher et al. 1998; Dressler et al. 1999; Postman et al. 1998, 2001). They have shown that star formation is suppressed as a consequence of the environment for those galaxies that have recently accreted (Dressler et al. 1999; Poggianti et al. 1999; Abraham et al. 1996; Balogh et al. 1997). These studies have clarified the clustercentric radial dependence of the spectral properties (Ellingson et al. 2001; Fisher et

${ }^{1}$ Based on observations made with the William Herschel Telescope operated on the island of La Palma by the Isaac Newton Group in the Spanish Observatorio del Roque de los Muchachos of the Instituto de Astrofisica de Canarias. 
Poggianti et al.

al. 1998; Balogh et al. 1997, 1998, 1999; Dressler et al. 1999), showing the potential of these observations to trace the infall history of clusters and constraining the evolution with redshift of the infall rate (Ellingson et al. 2001). Several of these studies have debated whether the star formation activity in clusters is enhanced in some of the infalling galaxies, before being finally quenched (Dressler et al. 1999; Balogh et al. 1999; Couch et al. 2001). Spectroscopic surveys of distant clusters have elucidated the star formation histories of the various Hubble types, underlining the lack of a one-to-one correlation between galaxy morphology and star formation properties (Dressler et al. 1999; Poggianti et al. 1999; Couch et al. 2001). They have compared the cluster and field spectroscopic properties at similar redshifts (Balogh et al. 1998; Dressler et al. 1999; Balogh et al. 1999; Postman et al. 2001) and those in clusters of different X-ray luminosities (Balogh et al. 2002). The first detailed comparison of cluster galaxy spectra across a redshift range is now being carried out (Tran et al. 2003).

However, none of these high-z spectroscopic studies have attempted a quantitative comparison with clusters in the local Universe. So far, the evolution inferred has always been based on the qualitatively well established result that the great majority of galaxies in the richest clusters today are devoid of any sign of current or recent star formation. The reason for this is that until now no similar study has been carried out on a suitable local dataset of analogous spectral quality sampling the global galaxy populations of clusters. Surprisingly, a complete and detailed census of the spectroscopic properties of galaxies in nearby clusters and groups is still lacking, but should soon be provided by the recent and ongoing surveys at low-z (e.g. Sloan, 2dF, Pimbblet et al. (2001); Fasano et al. (2003)).

In the last few years we have been carrying out a photometric and spectroscopic study of galaxies in the Coma cluster, obtaining deep wide-area B and R-band photometry and multifiber spectra for about 300 cluster members. The spectroscopic survey extends over more than 6 magnitudes, including both giant and dwarf galaxies down to $M_{B} \sim-14$. This is Paper VIII of a series presenting the results of these observations. The photometric (Komiyama et al. 2002) and spectroscopic (Mobasher et al. 2001) datasets were used to study the stellar population ages and metallicities as a function of galaxy magnitude and Hubble type (Poggianti et al. 2001a, 2001b) and of environment (Carter et al. 2002), to investigate the dynamics of the dwarf and giant populations (Edwards et al. 2002), and to derive the cluster luminosity function (Mobasher et al. 2003).

In this paper, we compare the spectroscopic properties of galaxies in Coma with those of galaxies in clusters at $z \sim 0.5$. The comparison of a single cluster with still relatively small samples of clusters at higher redshift is obviously limited. Nevertheless, we will argue that the gross spectral differences between the two epochs are more outstanding than the variance among the different distant clusters, and that this comparison is teaching us something about how the star formation properties of galaxies in clusters have evolved with redshift. After a brief description of our dataset ( $(2)$, we reanalyze our spectra in a similar fashion to what was done for the distant cluster data $(\S 3)$. The comparison of the spectral characteristics of luminous galaxies in Coma and in clusters at higher redshifts is presented in $\S 4$. In $\S 5$ we extend the spectroscopic analysis to galaxies at magnitudes fainter than can be probed at high redshifts, and find a significant population of poststarburst dwarfs. Their spectra and colors, incidence at different magnitudes, radial velocities, location within the cluster and positions relative to the substructure traced by the hot X-ray gas are presented in $\S 5$. In $\S 6$ we discuss the similarities and differences between the results presented here and previous work on Balmer-enhanced galaxies in Coma. Finally, in $\S 7$ we conclude with a discussion of the possible interpretation of our results within the current debate on environmental effects on star formation in galaxies.

In the following we adopt $H_{0}=65 \mathrm{~km} \mathrm{~s}^{-1} \mathrm{Mpc}^{-1}$ and a distance modulus to Coma of 35.16.

\section{OBSERVATIONS}

We have carried out a spectroscopic survey of faint and bright galaxies in two fields, each of size $32.5 \times$ $50.8 \operatorname{arcmin}^{2}$, in the direction of the center of the Coma cluster (Coma1) where the two dominant galaxies lie (NGC4874 and NGC4889), and in the south-west region where the cD galaxy NGC4839 is located (Coma3). These areas were imaged in the B and $\mathrm{R}$ bands with the Japanese mosaic CCD camera on the William Herschel Telescope (Komiyama et al. 2002, Paper I). A full description of the selection of the spectroscopic targets, observations and data reduction can be found in Mobasher et al. (2001, Paper II). Here we only summarize the most important characteristics of the sample and the data.

The spectra were taken with the WYFFOS multi-fibre spectrograph at the William Herschel Telescope. They are centered on $5100 \AA$ and extend over more than $3000 \AA$ with a resolution $6-9 \AA$ FWHM. Each fibre has a diameter of 2.7 arcsec, thus the spectra are sampling the central $1.3 \mathrm{kpc}$ of each galaxy.

Targets for spectroscopy were selected from our R-band magnitude limited sample with essentially no additional color or morphological selection criteria, except for the exclusion of a few ( 2 in Coma1 and 9 in Coma3, at most) extremely blue $(B-R<1)$ faint galaxies for which no cluster membership information is available. In the following we restrict our analysis to galaxies with velocities in the range $4000<v<10000 \mathrm{~km} \mathrm{~s}^{-1}$ (roughly corresponding to $3 \sigma$ cuts), which are considered members of the Coma cluster. With this membership criterion, the spectroscopic catalog comprises a total of 278 galaxies, of which 189 are in Coma1 and 89 in Coma3.

The survey extends over more than 6 magnitudes in galaxy luminosity, corresponding to a $\mathrm{B}$ band absolute magnitude in the range $M_{B} \sim-20.5$ to -14 mag for our choice of distance modulus to Coma. For convenience, in the following we will refer to "dwarf" and "giant" galaxies adopting a threshold $R_{3 K r}=16.3^{2}$ corresponding approximately to $M_{B}=-17.3$. This magnitude limit is expected to separate dwarf galaxies and low-luminosity ellipticals from spiral galaxies and giant ellipticals in most cases. Nevertheless, we stress that this division should only be

2 Magnitude over an aperture of radius 3 times the Kron radius. Throughout the paper, $R=R_{3 K r}$. 
considered a way to separate the bright and the faint subsamples and does not provide information about any other galactic characteristic such as morphological type or central surface brightness. According to our adopted division, the spectroscopic catalog comprises 160 dwarf and 118 giant galaxies members of the Coma cluster. In the following we further restrict the sample to galaxies brighter than $R<19$ because at magnitudes fainter than this the signalto-noise of the spectra is generally too low for a reliable spectroscopic analysis $(\S 3)$. This leaves us with a total of 147 dwarf and 113 giant Coma galaxies.

\section{LINE MEASUREMENTS AND CLASSIFICATION SCHEME}

In previous papers of this series we have used absorption line indices of the Lick system to estimate luminosityweighted ages and metallicities (Poggianti et al. 2001a). For the purposes of this paper, instead, we classify our Coma spectra into a number of spectral classes according to the method used for distant clusters presented in Dressler et al. (1999, MORPHS collaboration). The MORPHS spectral scheme allows a direct comparison between our Coma results and higher redshift clusters, since no comprehensive dataset of Lick indices is available yet for distant cluster galaxies.

The equivalent width of $\mathrm{H} \delta$ in absorption and of the main emission lines (if present: [OII] $\lambda 3727, \mathrm{H} \beta$, [OIII] $\lambda 4959,[\mathrm{OIII}] \lambda 5007, \mathrm{H} \alpha$ ) were measured as ratio of the line and the continuum fluxes, in the same way as was done for the MORPHS dataset (Dressler et al. (1999)), after binning the spectra at $6 \AA$ to match the same rest frame wavelength resolution of the spectra at $z=0.5$. The classification criteria are based only on the presence or absence of emission lines and on the strength of the $\mathrm{H} \delta$ line, which is commonly in absorption. These lines are good indicators of current (emission lines, especially [OII]) and recent $(\mathrm{H} \delta)$ star formation and the scheme is a convenient, relatively simple method to identify the broad star formation properties of post-starburst/post-starforming, starforming (spiral-like or starbursting) and passive galaxies. A description of the modeling and interpretation of each spectral class can be found in Poggianti et al. (1999). In the following we will consider three spectral types:

$k+a / a+k$ spectrum, if no emission-line is detected and $\mathrm{EW}(\mathrm{H} \delta)>3 \AA$. It is the spectrum of a poststarburst/post-starforming galaxy with no current star formation activity which was forming stars at a vigorous rate in the recent past (last $1.5 \mathrm{Gyr}$ ).

emission-line spectrum, if at least one emission-line is detected. It is the spectrum of a galaxy with some ongoing star formation or/and AGN activity. This activity can be at a low, moderate or high level. In the MORPHS scheme emission-line galaxies are further split into three different classes, while here we adopt a simplified scheme and group them all together.

$k$ spectrum, when no emission-line is detected and $\mathrm{EW}(\mathrm{H} \delta)<3 \AA$. This is the spectrum of a "passive" galaxy with neither on-going nor strong recent star formation during the last 1.5 Gyr.

We note that our spectra include either the wavelength region with [OII]3727 or the region around $\mathrm{H} \alpha$. In all cases, spectra were searched for other emission lines $(\mathrm{H} \beta$, [OIII] $\lambda 4959 / \lambda 5007)$. No emission line is detected in our $\mathrm{k}+\mathrm{a}$ and $\mathrm{k}$ spectra down to a few $\AA \mathrm{EW}(<2-5 \AA)$, which is the typical limit for detection. Even if some very weak emission were present and undetected, $\mathrm{k}+\mathrm{a}$ spectra do not resemble those of normal galaxies of any Hubble type. In fact, spectra with $\mathrm{EW}(\mathrm{H} \delta)>3 \AA$ and emission lines weaker than a few Angstrom are exceptional in samples of nearby field galaxies (see discussion in Poggianti et al. 1999, based on Kennicutt 1992, and Jansen et al. 2000, Jansen 2002 private communication). Moreover, normal, non-interacting galaxies of any morphological type and any emission-line strength typically have $\mathrm{EW}(\mathrm{H} \delta)$ smaller than 4-5 $\AA$ (Poggianti et al. 1999). As we will show later, about half of the $\mathrm{k}+\mathrm{a}$ galaxies we identify in Coma have $\mathrm{EW}(\mathrm{H} \delta)$ even higher than $5 \AA$.

\section{COMPARISON WITH DISTANT CLUSTERS}

In this section we carry out a comparison between the spectral types of distant cluster galaxies and those of Coma galaxies of similar absolute magnitudes. The Coma dataset is our spectroscopic survey described in $\S 2$. The distant dataset is the spectroscopic catalog of the MORPHS collaboration (Dressler et al. 1999), that includes 10 rich clusters at $\mathrm{z}=0.4-0.5$ with a wide range of properties in terms of concentration, optical and X-ray luminosity.

The MORPHS spectroscopic catalog has a luminosity distribution representative of cluster galaxies with an absolute $\mathrm{V}$ magnitude $M_{V} \leq-19.9$. Therefore, in comparing with the distant clusters, we first adopt an R-band luminosity cut in Coma $(R \sim 14.75)$ corresponding to $M_{V}<-19.9 .^{3}$ The spectral characteristics of fainter Coma galaxies will be discussed in the next section. No color or morphological selection criteria were applied in the original selection of the MORPHS galaxies. The small morphological bias (tendency for late-type galaxies to be slightly overrepresented) that was detected a posteriori was corrected for in Poggianti et al. (1999), thus the MORPHS numbers used here can be considered effectively drawn from a simply magnitude limited sample, and can be compared with our Coma data. Since on average the characteristics of galaxy spectra vary strongly with clustercentric radius, we only consider galaxies within a similar cluster area in all clusters, i.e. the central $\sim 1.4 \mathrm{Mpc}$.

The most remarkable difference we find between the MORPHS dataset and Coma is the fact that a large number of $\mathrm{k}+\mathrm{a}$ spectra were found in the MORPHS sample (about $20 \%$ of the total galaxy population), while this fraction is equal to zero (no galaxy detected) at comparable luminosities in Coma. No $\mathrm{k}+\mathrm{a}$ galaxy as luminous as those in the MORPHS sample is detected even outside the central 1.4 Mpc of Coma, i.e. in the South-West Coma3 region with NGC 4839, as further discussed in $\S 6$.

To assess the influence of variation in cluster properties, we can limit the comparison to the two MORPHS clusters whose X-ray luminosities more closely resemble Coma, $(\mathrm{Cl0016}+16 \text { and } 3 \mathrm{C} 295)^{4}$. These two clusters have even

\footnotetext{
3 This conversion in magnitudes assumes the rest-frame color of an Sbc galaxy $(\mathrm{V}-\mathrm{R}=0.5)$. This is a conservative choice because if the color of an elliptical was adopted, the $\mathrm{R}$ band limit in Coma would be even brighter (14.5).

4 The X-ray luminosity of Coma corresponds to $L_{X} \sim 9.510^{44} \mathrm{ergs} \mathrm{s}^{-1}$ when observed at $\mathrm{z}=0.5$ at $0.3-3.5 \mathrm{keV}$ and lies in between that of
} 
Poggianti et al.

higher $\mathrm{k}+\mathrm{a}$ fractions $(\sim 30 \pm 10 \%)$ than the whole sample, making the lack of bright $\mathrm{k}+\mathrm{a}$ galaxies in Coma even more striking.

Neither the uncertainties in the conversion between observed and absolute magnitudes (0.3 mag at most, due to the cosmological parameters and the galaxy colors adopted), nor a plausible small luminosity brightening in $M^{\star}$ at higher redshifts $(\sim 0.3 \mathrm{mag}$ in $\mathrm{V}$ in ellipticals between $\mathrm{z}=0.55$ and $\mathrm{z}=0$, (Smail et al. 1997b)) can come even close to explaining the lack of luminous $\mathrm{k}+\mathrm{a}$ 's in Coma. As we will see in the next section, in Coma we need to go two magnitudes fainter to find a significant $\mathrm{k}+\mathrm{a}$ population.

Numerous other spectroscopic surveys have found significant populations of $\mathrm{k}+\mathrm{a}$ galaxies in distant clusters (see for example Couch and Sharples (1987); Abraham et al. (1996); Dressler \& Gunn (1992); Fisher et al. (1998); Tran et al. (2003)). We have chosen to compare with the MORPHS sample because it is currently the largest sample of clusters at $z>0.3$ with detailed spectroscopic analysis, and because it is possible to adopt similar magnitude and area cuts, as well as line measurement methods, for our Coma data. We note however that much lower (but not zero) $\mathrm{k}+\mathrm{a}$ fractions than those found in the MORPHS clusters were detected in the cluster sample of the Canadian Network for Observational Cosmology by Balogh et al. (1999), even at redshifts comparable to the MORPHS sample. The mean incidence of $\mathrm{k}+\mathrm{a}$ 's in clusters and its variance at any cosmological epoch will be fully clarified by the ongoing and future large surveys of high redshift clusters. Currently, the contrast between the presence/absence of notable numbers of luminous $\mathrm{k}+\mathrm{a}$ galaxies in clusters at $\mathrm{z}=0.5 /$ Coma is remarkable and points to a significant evolution with redshift of this type of galaxy population.

Turning to emission-line galaxies, there is a tendency for these to be less common in Coma $(9.4 \pm 5 \%)$ than in the overall MORPHS sample $(26.5 \pm 3 \%)$. Choosing to compare only to clusters with similar X-ray luminosities, however, strongly attenuates the differences with Coma, since Cl0016+16 and 3C295 have emission-line fractions of $11.1 \pm 6 \%$ and $14.7 \pm 8 \%$, respectively. Thus, while the $\mathrm{k}+\mathrm{a}$ incidence does not depend much on cluster properties in the MORPHS sample, the emission-line fraction strongly does, which makes it hard to disentangle a reliable evolutionary trend of the emission-line fraction from a variance as a function of the cluster properties on the basis of the two datasets we consider here. Finally, k-type spectra dominate the Coma cluster representing $90.6 \pm 17 \%$ in our sample, compared to $\sim 51 \pm 4 \%$ in the distant cluster galaxies, and $\sim 55 \pm 15 \%$ in Cl0016+16 and 3C 295. Before presenting the spectroscopic properties of Coma galaxies fainter than $M_{V} \sim-20$ in $\S 5$, we first discuss the possible effects of the spectroscopic aperture on our spectral classifications and the implications for the conclusions reached so far.

\subsection{Radial color gradients in galaxies and aperture effects}

A reason for concern when comparing nearby and distant clusters is the difference in the galactic area (in $\mathrm{kpc}$ ) covered by the spectra at low and high redshift. The fibre diameter of our Coma spectra corresponds to the central $1.3 \mathrm{kpc}$ of the galaxies, while the slit spectrum at $\mathrm{z}=0.5$ samples $\sim 7 \times 18 \mathrm{kpc}$. Therefore, in principle, if the central regions of galaxies were older than the outer regions, the higher fraction of passive (k-type) galaxies in Coma compared to distant clusters and the lack of $\mathrm{k}+\mathrm{a}$ spectra could be due to aperture effects.

The star formation gradients observed in cluster galaxies actually go in the opposite direction. A number of studies have found evidence that the most recent episode of star formation tends to be more centrally concentrated in cluster galaxies than in field samples, both in starforming and post-starforming galaxies (Moss \& Whittle 1993; Koopmann \& Kenney 1998; Moss \& Whittle 2000; Rose et al. 2001; Bartholomew et al. 2001; Koopmann \& Kenney 2003). If this is generally the case, aperture effects would tend to underestimate the evolution with redshift.

However, we prefer to evaluate the impact of aperture effects on a galaxy-by-galaxy basis from our own data, employing radial color gradients within individual galaxies in Coma. Here we use the fact that color and spectral type are largely correlated and that if recent or ongoing star formation occurred in the outer regions of the galaxy (those not encircled within the fibre), these would tend to be bluer than the galaxy centre.

The B-R colors over an aperture equal to the fibre diameter $(2.7$ arcsec) are plotted against the total (3 Kron radii) colors in Fig. 1. For the comparison with the distant clusters, only the k-type galaxies with $R<14.75$ (left panel, filled dots) are relevant. All these luminous k-type galaxies have colors $(B-R)_{2.7^{\prime \prime}}>1.4$, and since in this case color and spectral type are derived from the same aperture, a color redder than 1.4 can be considered to correspond to a k-type spectrum. If the outer regions of a galaxy were significantly younger and displayed a more "active" spectral type, then its total color should be significantly bluer than 1.4, by more than $0.1 \mathrm{mag}$, as expected from the color-magnitude diagram in Fig. 2 and the colors of galaxies with various spectral types. This is obvious for the $\mathrm{k}$ versus emission-line types, but it is a solid argument also for $\mathrm{k}+\mathrm{a}$ 's. In fact, as can be seen also in Fig. 2, k+a galaxies can have a wide range in color, from blue "young" $\mathrm{k}+\mathrm{a}$ 's (observed soon after the termination of star formation) to "old" ones as red as ellipticals (after about 1-1.5 Gyr from truncation) (Couch and Sharples 1987). Although a lack of a color gradient therefore does not guarantee the absence of a red, old $\mathrm{k}+\mathrm{a}$ stellar population in the outskirts of the galaxy, if some of the bright k-classified galaxies had $\mathrm{k}+\mathrm{a}$ stellar populations outside of the fibre, statistically some of them (the young ones) should show a bluing at increasing distance from the galaxy center. We note that metallicity gradients will alter the color gradients as well, but their effects will be much more subtle than the large color differences implied by the coarse spectral typing adopted here.

Thus, a galaxy with an old centre and young outskirts should lie in Fig. 1 at $(B-R)_{\text {tot }}<1.4$ and $(B-R)_{2.7^{\prime \prime}}>$ 1.4; however, no significant population with these characteristics is observed, with at most one or two bright galaxies showing evidence for a gradient of this kind. This reinforces the conclusion based on age gradients from other

Cl0016+16 $\left(L_{X} \sim 11.810^{44} \mathrm{ergs} \mathrm{s}^{-1}, 0.3-3.5 \mathrm{keV}\right)$ and that of $3 \mathrm{C} 295\left(L_{X} \sim 6.410^{44} \mathrm{ergs} \mathrm{s}^{-1}, 0.3-3.5 \mathrm{keV}\right)$ (Smail et al. 1997a). 
studies that aperture effects are not likely to be responsible for the gross differences in spectral properties between Coma and the high redshift sample. We conclude that the observed spectral differences are real and that Coma has proportionally many more passive galaxies than the distant clusters, while lacking a significant population of luminous $\mathrm{k}+\mathrm{a}$ 's.

\section{EXPLORING LOWER LUMINOSITIES}

Any comparison with the distant cluster data is inevitably limited to the bright end of the galaxy luminosity function, while in nearby clusters it is possible to study also fainter galaxies. In Coma, our spectroscopic survey extends over more than 6 magnitudes down to $M_{B} \sim-14$. In the following, we limit our discussion to $R<19\left(M_{B}<-14.6\right)$ because at magnitudes fainter than this limit a large fraction of the galaxies have an uncertain spectral type due to the low signal-to-noise of the spectra.

In the previous section we showed that Coma lacks the population of luminous $\mathrm{k}+\mathrm{a}$ galaxies that is present in clusters at $\mathrm{z}=0.4$. Interestingly, very clear examples of $\mathrm{k}+\mathrm{a}$ spectra are found instead among the faint Coma galaxies.

Figure 3 presents the spectra of all our "secure" $\mathrm{k}+\mathrm{a}$ galaxies, with good $\mathrm{H} \delta$ measurements confirmed by the strength of other Balmer lines. The mean signal-to-noise of these spectra is 11.4/pixel, ranging from a maximum of $17 /$ pixel $(\# 90085)$ to a minimum of $6 /$ pixel $(\# 48397)^{5}$.

The $\mathrm{k}+\mathrm{a}$ spectra can be contrasted with a k-type spectrum in Fig. 4. We have chosen to show the $\mathrm{k}$ spectrum of a single galaxy with a magnitude similar to the $\mathrm{k}+\mathrm{a}$ spectra, instead of coadding several spectra into a "representative k-type" spectrum, because in this way the $\mathrm{S} / \mathrm{N}$ is comparable to that of the $\mathrm{k}+\mathrm{a}$ galaxies in Fig. 3. The difference in $\mathrm{H} \delta$ and other Balmer line strengths between the $\mathrm{k}$ spectrum and the $\mathrm{k}+\mathrm{a}$ 's is striking even from a simple visual inspection of the spectra. This is quantified by the measurements of the $\mathrm{EW}(\mathrm{H} \delta)$ in Table 1 . The table lists the galaxy identification number in our catalog, identification number in the catalog of Godwin et al. (1983), coordinates, $\mathrm{R}$ magnitude, B-R color and $\mathrm{H} \delta$ strength of the 13 secure $\mathrm{k}+\mathrm{a}$ galaxies in our sample and of other 10 more uncertain but likely $\mathrm{k}+\mathrm{a}$ 's, these latter being marked with a colon (":") in column 6 . The $\mathrm{k}+\mathrm{a}$ spectra cover a range of $\mathrm{H} \delta$ strengths, from very strong, text-book cases of post-starburst galaxies such as \#28211, \#30153 and \#31086 to weaker cases with $\mathrm{EW}(\mathrm{H} \delta)$ just above $3 \AA$, such as \#90085 and \#47098, whose line is just strong enough to belong to the $\mathrm{k}+\mathrm{a}$ class.

Magnitude distributions and color-magnitude diagrams of $\mathrm{k}, \mathrm{k}+\mathrm{a}$ and emission-line galaxies are presented in Fig. 2. Two aspects of this plot are remarkable. First, Coma $\mathrm{k}+\mathrm{a}$ 's in our sample are typically fainter than $M_{V} \sim$ -18.6 , or $R \sim 16$, i.e. more than about 3 magnitudes fainter than $M^{\star}$. The majority of them are classified as "dwarfs" according to our adopted definitions $(R>16.3)$, with the exception of two and three "giant" galaxies in the Coma3 and Coma1 fields, respectively. As pointed out in $\S 2$, the adopted dwarfs/giants division is somewhat arbitrary and is expected to only roughly separate dwarf galaxies and low-luminosity ellipticals from spiral galaxies and giant ellipticals. In the Coma1 field, the magnitude distribution of $\mathrm{k}+\mathrm{a}$ 's seems to cluster around a "characteristic luminosity" $\left(M_{V} \sim-18 /-17.5\right)$ (Fig. 2$)$, but it is hard to establish whether the lack of very faint $\mathrm{k}+\mathrm{a}$ 's is simply due to increasingly poor $\mathrm{S} / \mathrm{N}$ spectra. The deficit of $\mathrm{k}+\mathrm{a}$ 's among the brightest galaxies is instead a firm result.

Second, in the color-magnitude diagram of Fig. 2 a group of blue and a group of red $\mathrm{k}+\mathrm{a}$ 's can be easily distinguished. These most likely correspond to "young" $\mathrm{k}+\mathrm{a}$ 's (observed soon after the termination of star formation) and to "old" ones (observed at a later stage of the evolution). In agreement with this interpretation and with results of models of $\mathrm{k}+\mathrm{a}$ spectra (e.g. Couch and Sharples (1987); Poggianti and Barbaro (1996)), the average $\mathrm{EW}(\mathrm{H} \delta)$ of the blue group is significantly stronger than that of the red group (Table 1 ).

An equivalent width $\mathrm{EW}(\mathrm{H} \delta)>5 \AA$ testifies that a starburst occurred in the galaxy before star formation was quenched, as a simple interruption of otherwise quiescent star formation activity produces a spectrum with weaker Balmer lines (e.g. Couch and Sharples (1987); Poggianti and Barbaro (1996)). Most of the blue $k+a$ 's, and a few of the red $\mathrm{k}+\mathrm{a}$ 's, have EWs stronger than $5 \AA$, and thus must truly be post-starburst galaxies.

Morphological classifications based on visual inspection of deep images are available from Beijersbergen (2003) for a subset of our $\mathrm{k}+\mathrm{a}$ sample: these are mostly disky galaxies with spiral or merger/peculiar (M/P) morphologies, two of them being classified as M/P, one as an Sc, two as Sa's and one as S0/a. Only one red $\mathrm{k}+\mathrm{a}$ has an available classification from Beijersbergen (2003) and it is an S0 galaxy. A visual inspection of our images (Komiyama et al. 2002) confirms that $\mathrm{k}+\mathrm{a}$ 's are an heterogeneous population spanning the whole range of morphological types from early to late types and mergers. $\mathrm{K}+\mathrm{a}$ spectra are found in galaxies of all Hubble types also in distant clusters, where the majority of $\mathrm{k}+\mathrm{a}$ 's are disky galaxies (Wirth et al. 1994, Couch et al. 1998, Dressler et al. 1999, Poggianti et al. 1999). However, we should remember that our $\mathrm{k}+\mathrm{a}$ 's are fainter than those found in the distant clusters and that most of them are dwarfs to which a classification early/late does not apply straightforwardly. In our images, most of the $\mathrm{k}+\mathrm{a}$ 's are found to have companions and/or signs of tidal interactions, and most companions are much smaller objects than the already small k+a's. It is an open question whether these small companions have some effect on, or are somehow related to the $\mathrm{k}+\mathrm{a}$ phenomenon. An automated assessment of the light profiles all our $\mathrm{k}+\mathrm{a}$ galaxies is provided by the measurements of the Sersic index $N$ presented in Paper I. With the exception of two blue $\mathrm{k}+\mathrm{a}$ 's compatible with a de Vaucouleurs's profile $(N=0.25)$, all the other blue and red k+a's have exponential or steeper profiles $(N \geq 0.5)$, as the great majority of all Coma galaxies of similar luminosities do. A more detailed analysis of $\mathrm{k}+\mathrm{a}$ and Coma dwarf morphologies in general will be the subject of a future work."

Estimates of luminosity-weighted metallicities are available for $\mathrm{k}+\mathrm{a}$ galaxies from our earlier analysis based on spectral indices of the Lick system. In Paper III we found a large metallicity scatter among galaxies at any given luminosity fainter than $\mathrm{R}=16$. This is true also for both blue

\footnotetext{
5 The $\mathrm{S} / \mathrm{N}$ has been measured as an average of the blue and red sides of the $\mathrm{H} \delta$ line.
} 
Poggianti et al.

and red $\mathrm{k}+\mathrm{a}$ 's, that have metallicities from above solar to $[\mathrm{M} / \mathrm{H}] \sim-1.5$, and also for k-type galaxies of similar luminosity. Hence, the $\mathrm{k}+\mathrm{a}$ galaxies do not stand out for their metal content with respect to the rest of the population of galaxies of similar luminosity, and share the broad range in metallicity of all faint Coma galaxies.

We now examine the overall incidence of the various spectral classes in the dwarf and giant subsamples separately. ${ }^{6}$ Numbers and fractions of galaxies of each spectral type are given in Tables 2 and 3 for the Coma1, Coma3 and Coma1+Coma3 regions. Table 3 shows that $\mathrm{k}+\mathrm{a}$ 's constitute a significant fraction (10-15\%) of the dwarf galaxy population at $M_{B}>-17.3$, being much more common among the "dwarfs" than among the "giants". If we only include galaxies with secure spectral classifications, the $\mathrm{k}+\mathrm{a}$ fraction among the dwarfs remains similar $(16 \pm 5 \%)$. It is of course unknown whether a $\mathrm{k}+\mathrm{a}$ population of dwarf galaxies exists also at higher redshift, and if they are more, equally or less frequent than at $\mathrm{z}=0$.

\subsection{K+a dwarfs and substructure}

In their spectroscopic survey of early-type galaxies in Coma, Caldwell et al. (1993) detected enhanced Balmer lines in absorption in a large fraction of the galaxies in the South-West region of Coma, mostly between the cluster centre and the secondary peak of the X-ray emission in the $\mathrm{SW}$, in a region that has not been extensively sampled in our survey. They found instead very few such galaxies in the central field of the cluster. A spatial segregation of galaxies with peculiar star formation signatures can obviously be useful in identifying substructure, and can serve to investigate the effects of cluster-group merging on the properties of galaxies.

In Figure 5 we show the projected position on the sky of galaxies of different spectral types in our sample. From a simple inspection of this figure, $\mathrm{k}+\mathrm{a}$ galaxies do not seem preferentially located in any of the regions we surveyed. As shown in Table 3, the $\mathrm{k}+\mathrm{a}$ fractions are similar within the errors in Coma1 and Coma3. However, those with the strongest $\mathrm{H} \delta$ EWs (the blue $\mathrm{k}+\mathrm{a}$ 's) are all situated in projection towards the Coma1 central field, though only one of them is in the central projected 25 arcmin from the two central galaxies. We will show later in this section that blue $\mathrm{k}+\mathrm{a}$ 's are not randomly distributed in the cluster, and that a strong correlation with substructure becomes evident from a deeper analysis.

Interestingly, the mean radial velocity of the group of blue $\mathrm{k}+\mathrm{a}$ 's is $8120 \pm 709 \mathrm{~km} \mathrm{~s}^{-1}$, with all but one at $v>7200 \mathrm{~km} \mathrm{~s}^{-1}$ (see Table 1). In contrast, both the red $\mathrm{k}+\mathrm{a}$ 's and all faint $\mathrm{k}$ galaxies with $R>16$ in Coma1 have much lower mean velocities: $6992 \pm 761$ and $6854 \pm 244 \mathrm{~km}$ $\mathrm{s}^{-1}$, respectively ${ }^{7}$. The hypothesis that the group of blue $\mathrm{k}+\mathrm{a}$ 's and the other two groups are drawn from the same parent velocity distribution is rejected by a KolmogorovSmirnov test at the $93.3 \%$ and $99.995 \%$, respectively.

The strength of the lines and the color of the blue k+a's indicate a halting of the star formation within the last 500
Myr and probably at some point during the last $\sim 5 \times 10^{7}$ to $3 \times 10^{8}$ yr. In most cases (those with $\mathrm{EW}(\mathrm{H} \delta)>5 \AA$ ) a strong starburst must have preceded the quenching of star formation (Poggianti et al. 1999; Poggianti and Barbaro 1996). In contrast, the typical time elapsed since the last star formation in the red $\mathrm{k}+\mathrm{a}$ 's will be in the range 700-1500 Myr. The fact that the youngest $\mathrm{k}+\mathrm{a}$ 's are a kinematically distinct population from the general relaxed dwarf population (k-types) and older $\mathrm{k}+\mathrm{a}$ 's suggests that their star formation history was recently quenched as a result of their first interaction with the main cluster body.

The blue $\mathrm{k}+\mathrm{a}$ galaxies have a relatively high velocity dispersion $\left(1250 \mathrm{~km} \mathrm{~s}^{-1}\right)$ indicating that probably they are not part of a single infalling bound galaxy group of relatively small mass compared to the whole Coma cluster. Their velocities and velocity dispersion suggest instead they could be part of a filament converging onto Coma.

Proof of this, and a suggestive clue about the possible physical mechanism responsible for the $\mathrm{k}+\mathrm{a}$ spectra, comes from the recent X-ray mosaic observations of Coma obtained with XMM-Newton. Neumann et al. (2003) have recently identified and discussed X-ray substructure by fitting a smooth profile (an elliptical beta model of a relaxed cluster) and subtracting it from the data. The residuals reveal several structures, that are shown as contours in Fig. 6: besides the well known NGC4839 South-West group, Neumann et al. (2003) identify a large residual to the West of the cluster centre ("Western structure" in Fig. 6) elongated along the North-South direction, and a filament-like structure South-East of the centre ("Eastern structure" in Fig. 6), elongated along the East-West direction. The temperature map shown in color in Fig. 6 sheds further light on the accretion history of Coma. Neumann et al. conclude that the region of high temperature observed between the Western structure and the Coma center is caused by the infall of this structure, either via compression or via shock waves. These authors consider the two maxima in the western structure to be likely the result of the disruption of a galaxy group during its infall, instead of two galaxy groups falling at the same time. In contrast, the South-Eastern structure is cooler than the mean cluster temperature and is associated with a low-mass galaxy group dominated by two large galaxies, NGC4911 and NGC4921. Based on the filamentary form of this structure, the same authors conclude it is observed during the infall process while being affected by ram pressure stripping close to the cluster centre.

The coincidence of the position of the strongest $\mathrm{k}+\mathrm{a}$ galaxies and the X-ray structures is striking. Four k+a's with $\mathrm{EW}(\mathrm{H} \delta)>5 \AA$ (green dots in Fig. 6) trace the edge of the Western structure towards the Coma centre. Another three are associated with the Eastern structure, all at its western boundary. One $\mathrm{k}+\mathrm{a}$ with uncertain classification (\#6750, $\mathrm{k}+\mathrm{a}$ : in Table 1 ) is at the location of a strong temperature gradient between a hot and a cool region, though Neumann et al. (2003) warn that such hot

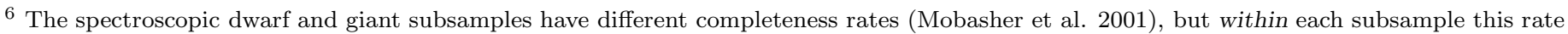

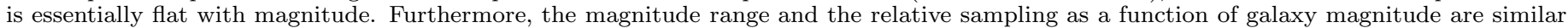
for the Coma1 and Coma3 spectroscopy. Hence, the relative proportions of spectral types in each subsample can be compared.

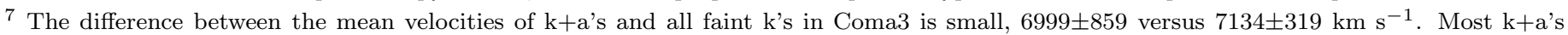

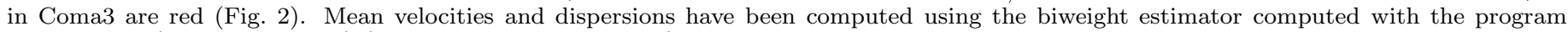
ROSTAT by (Beers et al. 1990) (see also Teague et al. 1990). 
spot could be a statistical artefact due to the low XMMNewton exposure time in that region.

The correlation between the location of the poststarburst galaxies and the substructure in the intracluster medium appears too strong to be a fortuitous coincidence. Young post-starbursts are distributed close to the edge of infalling substructures. In the case of the Western substructure this edge is the infalling front, while for the Eastern substructure it is unclear whether the group is moving to the West, as suggested by the appearance of the X-ray residuals, or to the East, as suggested by the positions of NGC4911 and NGC4921 (Neumann et al. 2003). Overall, this strongly suggests that the $\mathrm{k}+\mathrm{a}$ spectra, i.e. the truncation of the star formation activity in these galaxies and possibly the previous starburst, could be the result of an interaction with the hot intracluster medium. Hence, as far as Coma $\mathrm{k}+\mathrm{a}$ galaxies are concerned, there is suggestive evidence that the origin of the $\mathrm{k}+\mathrm{a}$ spectrum is a clusterrelated and, in particular, an ICM-related phenomenon that is closely connected with the dynamical state of the cluster. We note that the $\mathrm{H} \delta$ strength of the blue $\mathrm{k}+\mathrm{a}$ galaxies implies that star formation was truncated in these galaxies on a short timescale, i.e. short compared to the $\mathrm{k}+\mathrm{a}$ timescale of 1-1.5 Gyr. In fact, a slowly declining star formation activity such as that envisaged if galaxies simply lost their gas halo reservoir when becoming part of a group ("strangulation", e.g. Bower \& Balogh 2003) does not produce such strong Balmer lines.

The two large galaxies that dominate the Eastern structure (NGC4911 and NGC4921) have velocities of 7973 and $7560 \mathrm{~km} / \mathrm{sec}$, respectively, hence they too have velocities significantly higher than the cluster median, as do the blue/strong $\mathrm{k}+\mathrm{a}$ 's. This is consistent with the hypothesis that the three strong $\mathrm{k}+\mathrm{a}$ 's close to the Eastern structure are physically associated with the NGC4911/4921 group. It remains to be interpreted why the other strong/blue $\mathrm{k}+\mathrm{a}$ 's, and especially those four along the Western structure, also have similar velocities, and whether the two structures are somehow associated and/or related to the NGC4839 group and on larger scales with the filament connecting Coma and A1367 along the Great Wall.

We stress here the importance of isolating the youngest $\mathrm{k}+\mathrm{a}$ galaxies using their blue colors and strong equivalent widths. The red $\mathrm{k}+\mathrm{a}$ phase has a timescale that is comparable to the core crossing time in a cluster like Coma, and any signature of the link between the truncation of star formation and the location within a substructure is thus erased in the older $\mathrm{k}+\mathrm{a}$ 's, while it is still detectable in the youngest subsample of $\mathrm{k}+\mathrm{a}$ 's.

Obviously, establishing a correlation between the star formation history of the $\mathrm{k}+\mathrm{a}$ galaxies and the substructure within Coma would have been impossible on the basis of simple location on the sky (Fig. 5), while this correlation appears evident once a detailed X-ray map reveals the complicated structure in the hot intracluster gas. On the other hand, by isolating the strongest/youngest poststarburst galaxies we have identified specific areas in the cluster where a physical mechanism must have been recently at work affecting the star formation activity of these galaxies.

\subsubsection{Emission-line galaxies}

Interestingly, the few emission-line galaxies in the Coma1 field (all starbursts or star-forming galaxies of unknown star formation intensity) are mostly found along the edge of the Western structure (Fig. 7), with the exception of \#15480 that is located just South of the Eastern structure. However, only two of the Coma1 emission-line galaxies lie at high velocities (7525 and $9443 \mathrm{~km} \mathrm{~s}^{-1}$, galaxies \#15480 and \#77938, respectively), while the other four have velocities between 5007 and $5666 \mathrm{~km} / \mathrm{sec}$. The two high velocity emission-line galaxies (both starbursting galaxies) could be associated with the same subsystems to which the majority of the blue $\mathrm{k}+\mathrm{a}$ galaxies belong. The other Coma1 emission-line galaxies remain a puzzle: their alignment with the X-ray substructure suggests they too could be related to the same infalling group, but their low velocities seem to contradict this hypothesis.

Emission-line galaxies are proportionally more numerous in the Coma3 than in the Coma1 field (Figs. 5 and 6 ). For dwarfs we estimate a $13 \%$ fraction of emission-line galaxies in Coma 3 versus 5\% in Coma1, and among giants $33 \%$ in Coma 3 versus 9\% in Coma1 (Table 3). It should be noted that the emission-line properties of dwarf and giant spectra are on average very different, because the former mostly present moderate or strong lines, while the latter tend to have very weak emission, sometimes barely detectable. Hence, we stress that the higher emission-line fractions among giants should not be considered as indicative of a generally higher star formation activity in brighter galaxies.

\subsection{Faint $k+a$ galaxies and aperture effects}

Similarly to what has been done in $\S 4.1$, we estimate the relevance of aperture effects in order to assess the reliability of the comparison between giants and dwarfs. More luminous galaxies are on average bigger in size and the fibre is sampling proportionally a smaller portion of the galaxy. Could the observed higher proportion of $\mathrm{k}+\mathrm{a}$ 's among dwarfs be caused by aperture effects? For this to be the case, the $\mathrm{k}+\mathrm{a}$ stellar populations should be preferentially located outside of the central region of the galaxy. As discussed also in $\S 4.1$, the latest star formation episode in cluster galaxies, and post-starburst/poststarforming galaxies in particular, is found to be instead centrally concentrated (Rose et al. (2001); Bartholomew et al. (2001), see also Norton et al. (2001) for field k+a's). Moreover, color gradients can again be used to evaluate stellar population gradients within galaxies. The left panel of Fig. 1 presents the central versus total color of all ktype galaxies, both brighter and fainter than $R=14.75$. The arguments discussed in $\S 4.1$ apply here as well: we do not observe a significant population of galaxies whose color gradients suggest a noticeable difference between the spectral type in the region covered by the fibre and the integrated spectral type, within the gross spectral classification scheme adopted here. Only very few galaxies have a total color that is significantly bluer than the central color and which corresponds to star-forming or blue poststarforming galaxies. Galaxies with a central k-type and an outer $\mathrm{k}+\mathrm{a}$ red population at a late stage of its evolution would go unnoticed in this analysis. Thus, in principle we cannot exclude the existence of giant galaxies with red $\mathrm{k}+\mathrm{a}$ spectra in their outer regions that went undetected in our 
data.

Central versus total colors of $\mathrm{k}+\mathrm{a}$ galaxies are shown in the right panel of Fig. 1. As discussed above, these tend to be generally faint galaxies, hence the fibre spectrum covers a significant fraction of the galactic area. Most of these galaxies have a negligible color difference between the central 2.7" and the outskirts, while the two galaxies with larger color gradients are bluer in the center.

\section{COMPARISON WITH PREVIOUS WORK ON COMA AND OTHER NEARBY CLUSTERS}

Though a low fraction of $\mathrm{k}+\mathrm{a}$ spectra among luminous galaxies in nearby clusters is often cited (Dressler 1987), a quantitative comparison of the incidence of this type of galaxy in nearby and in distant clusters has not been carried out to date, due to the lack of large samples of galaxy spectra in low redshift clusters suitable for this purpose. This is the first time that such a comparison is attempted.

Spectra with enhanced Balmer lines in absorption have been previously reported in early-type galaxies in Coma and other nearby clusters in a number of papers by Caldwell, Rose and collaborators (e.g. Rose et al. 2001 and references therein). Out of the 12 galaxies in the sample of Balmer-enhanced galaxies without emission studied by Caldwell et al. (1993) (hereafter C93), six are in common with our Coma spectroscopic survey. The comparison of the results is rather instructive and shows that our $\mathrm{k}+\mathrm{a}$ galaxies and their Balmer-enhanced galaxies have on average significantly different Balmer line intensities. In fact, only 1 out of the 6 galaxies in common qualifies to be classified as $\mathrm{k}+\mathrm{a}$ in our spectra ${ }^{8}$, while the other five galaxies have $\mathrm{EW}(\mathrm{H} \delta)<3 \AA$ in our data. However, 4 of these galaxies (D21, D146, D127, D14), although with a k-type spectrum, do show signs of recent star formation based on the analysis of age- and metallicity-sensitive line indices of the Lick system measured in our spectra (Poggianti et al. 2001a), in agreement with Caldwell et al.'s detection of recent activity. ${ }^{9}$. In these galaxies the latest star formation episode must have been weaker or is older than in galaxies with a $\mathrm{k}+\mathrm{a}$ spectrum, but it can still be detected by a spectral analysis that is sensitive to even weaker/older activity, such as that performed by Caldwell and collaborators and by us in Poggianti et al. (2001a). ${ }^{10}$ This is in a sense expected since the Caldwell et al. sample is composed only of morphologically selected early-type galaxies, while ours is a magnitude limited sample with no color or morphological selection criteria and, as discussed in $\S 5$, the strongest $\mathrm{k}+\mathrm{a}$ galaxies turn out to have late-type and merger/peculiar morphologies. Their sample is therefore probably more skewed towards galaxies at a later stage in their path to become more passive/earlier-type galaxies. In fact, Caldwell et al. (1993) note that these galaxies are similar to the red $\mathrm{H} \delta$ strong galaxies in distant clusters, i.e. those observed at a late stage of their Balmer-strong phase. The lower line/burst strength in their Balmer-enhanced galaxies as compared to distant clusters was also pointed out by Caldwell \& Rose (1997) for Coma and other four nearby clusters. It is interesting to note that Caldwell et al. (1993) also stressed that the majority of their Balmerenhanced galaxies are in a luminosity range unexplored in distant clusters because they are too faint.

A main result from Caldwell et al. (1993) was that the Balmer-enhanced galaxies were mostly located in a region South-West of the center of Coma, where the incidence of such galaxies was much higher than in the central region of the cluster. However, even fainter galaxies with recent star formation were not found preferentially in the SW region, but spread all over, also to the NE of the central galaxy (Caldwell \& Rose 1998). The same is true for the work of Castander et al. (2001), who found five post-starburst galaxies out of 196 Coma members with no apparent correlation with the NGC 4839 group.

To summarize, while the $\mathrm{k}+\mathrm{a}$ galaxies we discuss in this paper are examples of Balmer-strong galaxies above a certain line-strength threshold and below a certain "age", Balmer-enhanced galaxies amply discussed in previous papers include also weaker/older cases and, on average, have weaker lines than $\mathrm{k}+\mathrm{a}$ 's. We suspect that these differences in spectral properties are related to the different selection strategy for our sample (which had no color or morphological selection criteria) compared to previous samples, which were limited to early-type morphologies. We also remind the reader that different spectral classification schemes were adopted: the $\mathrm{EW}$ of $\mathrm{H} \delta$ in our study versus the $\mathrm{CN} / \mathrm{H} 8$ index (a fit to the slope of the spectrum between 3858 and $3893 \AA$ ) for Balmer-enhanced galaxies in Caldwell et al. (1993). However, both our $\mathrm{k}+\mathrm{a}$ 's and the Balmer-enhanced spectra identified by others are found in galaxies fainter than the $\mathrm{k}+\mathrm{a}$ 's observed at high redshift. The regions sampled in this work and in Caldwell et al. (1993) only partly overlap in the South-West region of the cluster, thus we are unable to confirm the excess of Balmer-enhanced spectra these authors found in that specific area. Within the regions we have sampled, $\mathrm{k}+\mathrm{a}$ galaxies apparently do not show a preferential spatial location in projection on the sky, but the group of youngest/strongest $\mathrm{k}+\mathrm{a}$ 's lie in projection towards the central region of Coma, though they avoid the cluster centre, and, as discussed in $\S 5$, their positions are closely related to X-ray substructure. In addition, they stand out for their high recession velocities compared to the cluster mean. Finally, this is the first time an attempt is made to quantify the incidence of $\mathrm{k}+\mathrm{a}$ spectra in the galaxy populations of Coma as a whole and as a function of galaxy luminosity, on a sample of galaxies selected irrespectively of morphology. Thus, no direct comparison regarding this can be made with other work.

\section{DISCUSSION}

The results presented in this paper raise one compelling question: why there are luminous $\mathrm{k}+\mathrm{a}$ 's in clusters at high

8 This is galaxy D216, \#90085 in this work. The C93 multifiber spectra cover an area only slightly smaller than ours (2"), and they use the CN/H8 index, which is a fit to the slope of the spectrum between 3858 and $3893 \AA$, to identify Balmer-enhanced galaxies.

9 There is only one case (galaxy D34) where our and Caldwell et al. 1993 results disagree because we do not detect any sign of star formation during the last 9 Gyrs, neither with the MORPHS method nor using the Lick system.

10 This is true also when comparing with the sample of Caldwell et al. (1998), where both their and our 2001 work identify galaxies with young (G2603), intermediate-age (G3205, G3126) and old (G2385, G3707) stellar populations, with an excellent agreement on the luminosity-weighted ages derived by the two studies. None of these galaxies shows a $\mathrm{k}+\mathrm{a}$ spectrum in our data. 
redshift while these are notably absent in Coma, where only fainter $\mathrm{k}+\mathrm{a}$ 's are observed?

Given the magnitude limits of high-z spectroscopic surveys, it is unknown how many faint $\mathrm{k}+\mathrm{a}$ 's exist in clusters at $\mathrm{z}=0.5$, as the luminous ones could simply be the tip of the iceberg. Trying to infer this indirectly from the spectroscopic ages of our Coma dwarfs is difficult due to the uncertainties involved, especially those regarding the evolution of the infall rate of galaxies onto Coma, the spectral type mix of the infalling population and the timescale for the halting of star formation once a star-forming galaxy enters a cluster.

In principle, as some of the physical mechanisms affecting galaxies in dense environments are expected to act differently depending on galaxy mass, the cause of the star formation truncation in luminous $\mathrm{k}+\mathrm{a}$ 's at high-z and in faint ones at low-z is not necessarily the same. However, those physical properties of clusters that are most relevant for their influence on galaxies (such as the effects of encounters with other cluster galaxies and the properties of the intracluster medium) have not evolved significantly between $\mathrm{z}=0.5$ and $\mathrm{z}=0$. Thus, environmental effects on galaxies of a given mass should be similar at the two epochs. It is therefore unlikely that changes in cluster physical conditions can explain the evolution in the luminous $\mathrm{k}+\mathrm{a}$ population. One cluster property that is expected to vary with redshift is the average amount of substructure, which should increase at higher redshift. Shocks and/or changes of the tidal gravitational field during group-cluster and cluster-cluster merging are expected to significantly affect star formation in galaxies (e.g. Bekki 1999). However, if the change with redshift in the average amount of substructure were the cause of the evolution of the luminous $\mathrm{k}+\mathrm{a}$ population, then luminous $\mathrm{k}+\mathrm{a}$ 's should also be found in merging clusters at low-z. There isn't any low redshift cluster to date with a reported high $\mathrm{k}+\mathrm{a}$ fraction among luminous galaxies. Large samples and a systematic investigation of the spectroscopic properties of galaxies in nearby clusters will further clarify this issue (Fasano et al. 2003).

More probably, the evolution in the incidence of luminous $\mathrm{k}+\mathrm{a}$ 's in clusters is due to an evolution with redshift in the properties of galaxies infalling into clusters, and reflects a cosmic "downsizing effect", for which some evidence has emerged in the last years: going to lower redshift, the maximum luminosity/mass of galaxies with significant star formation activity seem to decrease, possibly both in clusters (Smail et al. 1998, Bower et al. 1999, Kodama \& Bower 2001, Poggianti et al. 2001a) and in the field (Cowie et al. 1996, Kauffmann et al. 2003). In fact, if star formation at higher $\mathrm{z}$ was active on average in more massive galaxies than at lower $z$, regardless of environment, and star formation is quenched as a consequence of the infall onto massive and dense environments, then the $\mathrm{k}+\mathrm{a}$ phenomenon should be conspicuous in luminous galaxies in distant clusters and insubstantial among luminous galaxies in nearby clusters, as is observed.

Even more intriguing is searching for the physical cause of the downsizing effect. There can be several possible reasons for the fact that star formation in low mass galaxies seems to be more protracted on average than in massive galaxies. This could simply be due to an evolution "in- trinsic" to galaxies. It has been suggested, for example, that the star formation efficiency could increase with dark matter halo mass, perhaps as a result of supernova feedback processes, resulting in the majority of massive galaxies not forming stars at the present day (Kauffmann et al. 2003). Star formation in faint galaxies is also expected to be inhibited by the UV background radiation after reionization $(z<7-12)$ and before the background dropped $(z<1)$, preventing rapid cooling in this period (Babul \& Rees 1992; Benson et al. 2002). This effect becomes progressively more important towards fainter galaxies, and can thus be responsible for a slower or suppressed early star formation in the dwarfs (Skillman et al. 2003), leaving a gas reservoir for later star formation. In this case, the cause of the downsizing effect would be external to galaxies (i.e. the background radiation field), but not necessarily related to the environment.

Or could the downsizing effect itself be an "environmental" effect? Recent studies have found a change in the star formation properties of galaxies at a relatively low critical threshold in local galaxy density, interpreting this result as evidence that suppression of star formation occurs in groups as a consequence of the environment (Kodama et al. 2001, Lewis et al. 2002, Gomez et al. 2003). Going to lower redshift, proportionally more galaxies infalling onto a rich cluster like Coma are expected to have resided and therefore to possibly have been "pre-processed" (=have their star formation activity switched off) in moderately massive environments such as groups or small clusters. If the growth of mass structure in the Universe had an important role in establishing the observed decline in star formation rate from $\mathrm{z}=2$ to the present (Bower \& Balogh 2003, and references therein), it could be responsible for a decline in the number of luminous star-forming galaxies available and, as a consequence, for the lack of luminous $\mathrm{k}+\mathrm{a}$ 's at low $\mathrm{z}$. However, if this effect worked independently of the galaxy mass, then we shouldn't be observing $\mathrm{k}+\mathrm{a}$ 's in Coma at all, even at low luminosities, and thus shouldn't observe a down-sizing effect. In fact, why would the star-forming progenitors of the faint $k+a$ 's not have been quenched/pre-processed as more luminous galaxies were? Less massive galaxies are expected to be actually more strongly disrupted by environmental influences than more massive galaxies, due to their smaller gravitational potential.

If the growth of structure had a significant impact on the evolution of the global cosmic star formation, in order for this scenario to be simultaneously responsible for the evolution of the luminous $\mathrm{k}+\mathrm{a}$ population and consistent with the downsizing effect, the environmental conditions acting on galaxies should vary with the galaxy mass. This could occur, for example, if on average dwarf galaxies infalling into clusters today have inhabited in the past significantly different (less massive) environments than infalling massive galaxies did. It will be interesting to investigate the history of merging halos using high-resolution simulations and explore how this depends on present-day galaxy mass.

Observing $\mathrm{k}+\mathrm{a}$ galaxies in Coma we are probably witnessing the transformation of late-type star-forming or starbursting dwarfs into dwarf spheroidals/ellipticals as a consequence of the cluster environment. Evidence for such a transformation from blue star-forming to red pas- 
sive dwarfs in clusters comes also from a comparison of the faint end of the luminosity function at $\mathrm{z}=0.2$ and $\mathrm{z}=0$ (Wilson et al. 1997). The blue $\mathrm{k}+\mathrm{a}$ 's are a case in which we directly observe this transformation process still ongoing in a nearby cluster.

Another puzzling question remains open at this point: why are most of the blue $\mathrm{k}+\mathrm{a}$ 's we observe in Coma truly post-starburst systems, as testified by the exceptionally strong Balmer lines? What triggered the starburst? Again, the nature versus nurture options lie open: was the burst an episode related to processes intrinsic to the galaxy itself, or was it induced by external, environmental effects? An intrinsic cause would mean that many of the unperturbed dwarfs unaffected by the cluster environment should be in a starburst phase, i.e. have experienced a discontinuous star formation history with recent periods of enhanced activity. Spectroscopic surveys of dwarfs in lower density environments should be able to provide the answer. If environment was instead the cause of the starburst, detailed studies of the group of blue $\mathrm{k}+\mathrm{a}$ 's (their morphologies, immediate surroundings, distribution of star formation activity and of neutral and molecular gas within the galaxy, stellar and gaseous kinematics etc.) might help in understanding what physical mechanism triggered the burst and, in particular, if it was the interaction with the intracluster medium as discussed in $\S 5$.

To conclude, it is possible that the kind of "activity" observed in distant clusters ( $\mathrm{k}+\mathrm{a}$ spectra, or recently terminated star formation) is still ongoing today as it was in the past, but is simply affecting galaxies with a different range of masses. This could reflect a change with redshift of the typical mass of a star-forming galaxy infalling onto a rich cluster. The evolution of this typical mass could be driven either by intrinsic or by "environmental" effects, i.e. by the previous "environments" experienced by the infalling galaxies throughout their evolution.

\section{SUMMARY}

In this paper we have presented a quantitative comparison between the spectroscopic properties of galaxies in Coma and those in rich clusters at $\mathrm{z}=0.5$. The main results can be summarized as follows:

a) Coma lacks the population of luminous $\left(M_{V} \leq-20\right)$ galaxies with post-starburst/post-starforming $(\mathrm{k}+\mathrm{a})$ spectra that is present in most distant cluster samples.

b) In contrast, a numerous population of $\mathrm{k}+\mathrm{a}$ galaxies is present in Coma at fainter magnitudes, representing a significant fraction of the cluster dwarfs. These faint $\mathrm{k}+\mathrm{a}$ 's clearly divide into a red and a blue group, probably in an advanced and an early stage of their $\mathrm{k}+\mathrm{a}$ phase, respec- tively. The blue k+a's are also those with the strongest Balmer lines in absorption and the great majority of them have recently undergone a strong starburst. These blue $\mathrm{k}+\mathrm{a}$ 's display spiral or merger/peculiar morphologies.

c) While the red $\mathrm{k}+\mathrm{a}$ 's are found both in the central region and in a region South-West of the centre, the blue $\mathrm{k}+\mathrm{a}$ 's are all found in projection towards the central 1.4 Mpc. Generally, k+a's are not observed towards the cluster centre. The radial velocities of the blue/strong $\mathrm{k}+\mathrm{a}$ 's are much higher than the cluster mean, indicating they are a kinematically distinct population from the general relaxed dwarf population. Their high velocity dispersion $\left(1250 \mathrm{~km} \mathrm{~s}^{-1}\right)$ argues against them being part of a single virialized infalling group and already suggests they could belong to a substructure converging onto Coma. A comparison with the X-ray residuals and temperature map shows that the young, strong post-starburst galaxies are located at the edges of two substructures, suggesting that interaction with the intracluster medium is the most likely cause of the interruption of the star formation and, possibly, of the previous starburst.

d) We have discussed our results in the context of previous work on Balmer-enhanced galaxies in Coma. The $\mathrm{k}+\mathrm{a}$ galaxies discussed here have on average stronger Balmer lines in absorption than Balmer-enhanced galaxies, and probably represent the youngest/strongest examples of a common phenomenon, and the closest local counterparts to the distant $\mathrm{k}+\mathrm{a}$ population.

e) The different incidence of luminous $\mathrm{k}+\mathrm{a}$ galaxies at $\mathrm{z}=0.5$ and $\mathrm{z}=0$ likely reflects an evolution in the properties of the infalling galaxies instead of a change in the physical mechanisms at work in clusters. We suggest that the lack of luminous $\mathrm{k}+\mathrm{a}$ 's in Coma and the presence of faint ones provides further evidence of a "downsizing effect": the maximum luminosity/mass of galaxies with significant star formation activity seems to decrease at lower redshifts. The physical origin of this downsizing effect is unknown. It could be driven by the intrinsic evolution of galaxies, or could be related to an "environmental effect" connected with the growth of structure in the Universe and the progressively more massive environments inhabited by galaxies as evolution proceeds. This latter is a viable scenario only if less massive galaxies infalling into clusters today inhabited in the past, on average, less dense/massive environments than infalling massive galaxies did.

We thank Andrea Biviano, Richard Bower, Erica Ellingson, Jim Rose, Ian Smail, Jacqueline van Gorkom and the anonymous referee for useful discussions and/or suggestions that helped improving this paper.

\section{REFERENCES}

Abraham, R.G., Smecker-Hane, T.A., Hutchings, J.B., Carlberg, R.G., Yee, H.K.C., Ellingson, E., Morris, S., Oke, J.B., Rigler, M., 1996, ApJ, 471, 694

Babul, A. \& Rees, M.J., 1992, MNRAS, 255, 346

Balogh, M.L., Morris, S.L., Yee, H.K.C., Carlberg, R.G., Ellingson, E., 1997, ApJ, 488, L75

Balogh, M.L., Schade, D., Morris, S.L., Yee, H. K. C., Carlberg, R. G., Ellingson, E., 1998, ApJ, 504, L75

Balogh, M.L., Morris, S.L., Yee, H. K. C., Carlberg, R. G., Ellingson, E., 1999, ApJ, 527, 54
Balogh, M.L., Bower, R.G., Smail, I., Ziegler, B.L., Davies, R.L., Gaztelu, A., Fritz, A., 2002, MNRAS, 337, 256

Bartholomew, L.J., Rose, J.A., Gaba, A.E., Caldwell, N., 2001, AnJ, 122,2913

Beers, T.C., Flynn, K., Gebhardt, K., 1990, AnJ, 100, 32

Beijersbergen, M., 2003, PhD Thesis, University of Groningen

Bekki, K., 1999, ApJ, 510, L15

Benson, A.J., Lacey, C.G., Baugh, C.M., Cole, S., Frenk, C.S., 2002, MNRAS, 333, 156 
Bower, R.G., Terlevich, A., Kodama, T., Caldwell, N., 1999, in Star Formation in Early Type Galaxies, ASP Conf. Ser. 163, eds. P. Carral \& J. Cepa, p. 211

Bower, R.G. \& Balogh, M.L., 2003 in Carnegie Observatories Astrophysics Series, Vol.3: Clusters of Galaxies: Probes of Cosmological Structure and Galaxy Evolution, eds. J.S. Mulchaey, A. Dressler and A. Oemler (Cambridge: Cambridge University Press)

Butcher, H., Oemler, A., Jr. 1984, ApJ, 285, 426

Caldwell, N., Rose, J.A., Sharples, R.M., Ellis, R.S., Bower, R.G., 1993, AnJ, 106, 473

Caldwell, N., Rose, J. A. 1997, AJ, 113, 492

Caldwell, N., Rose, J. A. 1998, AJ, 115, 1423

Carter, D., Mobasher, B., Bridges, T.J., Poggianti, B.M., Komiyama, Y., Kashikawa, N., Doi, M., Iye, M., Okamura, S., Sekiguchi, M., Shimasaku, K., Yagi, M., Yasuda, N., 2002, ApJ, 567, 772

Castander, F.J., Nichol, R.C., Merrelli, A. et al., 2001, AnJ, 121, 2331

Couch, W.J., Sharples, R.M., 1987, MNRAS, 229, 423

Couch, W. J., Balogh, M.L., Bower, R. G., Smail, I., Glazebrook, K., Taylor, M. 2001, ApJ, 549, 820

Cowie, L.L., Songaila, A., Hu, E.M., Cohen, J.G., 1996, AnJ, 112, 839

Dressler, A., 1987, in Nearly normal galaxies: From the Planck time to the present, New York, Springer-Verlag, p. 276

Dressler, A., Oemler, A., Jr., Couch, W. J., Smail, I., Ellis, R. S., Barger, A., Butcher, H., Poggianti, B. M., Sharples, Ray M. 1997, ApJ, 490, 577

Dressler, A., Gunn, J.E., 1992, ApJS, 78, 1

Dressler, A., Smail, I., Poggianti, B. M., Butcher, H., Couch, W., Ellis, R., Oemler, A., 1999, ApJS, 122, 51

Edwards, S.A., Colless, M., Bridges, T.J., Carter, D., Mobasher, B., Poggianti, B.M., 2002, ApJ, 567, 178

Ellingson, E., Lin, H., Yee, H. K. C., Carlberg, R. G. 2001, ApJ, 547, 609

Fasano, G., Poggianti, B.M., Couch, W. J., Bettoni, D., Kjærgaard, P., Moles, M., 2000, ApJ, 542, 673

Fasano, G., Poggianti, B.M., Bettoni, D., Pignatelli, E., Marmo, C., Moles, M., Kjærgaard, P., Varela, J., Couch, W., Dressler, A., 2003, MemSAIt, 74, 355 (also astro-ph 0210172)

Fisher, D., Fabricant, D., Franx, M., van Dokkum, P., 1998, ApJ, 498, 195

Godwin, J.G., Metcalfe, N., Peach, J.V., 1983, MNRAS, 202, 113

Gomez, P.L., Nichol, R.C., Miller, C.J. et al., 2003, ApJ, 584, 210

Jansen, R.A., Fabricant, D., Franx, M., Caldwell, N., 2000, ApJS, 126,331

Kauffmann, G., Heckman, T.M., White, S.D.M. et al., 2003, MNRAS, 341, 54

Kelson, D.D., van Dokkum, P.G., Franx, M., Illingworth, G.D., Fabricant, D., 1997, ApJ, 478, L13

Kelson, D.D., Illingworth, G.D., van Dokkum, P.G., Franx, M., 2000, ApJ, 531, 184

Kennicutt, R.C., 1992, ApJS, 79, 255

Kodama, T., Arimoto, N., Barger, A.J., Aragon-Salamanca, A., 1998, A\&A, 334, 99

Kodama, T., Bower, R.G., 2001, MNRAS, 321, 18
Kodama, T., Smail, I., Nakata, F., Okamura, S., Bower, R.G., 2001, ApJL, 562, L9

Komiyama, Y., Sekiguchi, M., Kashikawa, N., et al., 2002, ApJS, 138, 265 (Paper I)

Koopmann, R., A., Kenney, J.D., 1998, ApJL, 497, L75

Koopmann, R., A., Kenney, J.D., 2003, in press (astro-ph 0209547)

Lewis, I., Balogh, M., De Propris, R. et al. 2002, MNRAS, 334, 673

Lubin, L.M., Oke, J.B., Postman, M., 2002, AJ, 124, 1905

Mobasher, B., Bridges, T.J., Carter, D., Poggianti, B. M., et al., 2001. ApJS, 137, 279 (Paper II)

Mobasher, B., Colless, M., Carter, D., et al., 2003, ApJ, 587, 605

Moss, C. \& Whittle, M., 1993, ApJ, 407, L17

Moss, C. \& Whittle, M., 2000, MNRAS, 317, 667

Neumann, D.M., Lumb, D.H., Pratt, G.W., Briel, U.G., 2003, A\&A, 400,811

Norton, S.A., Gebhardt, K., Zabludoff, A.I., Zaritcky, D., 2001, ApJ, 557,150

Pimbblet, K.A., Smail, I., Edge, A.C., Couch, W.J., O'Hely, E., Zabludoff, A.I., 2001, MNRAS, 327, 588

Poggianti, B.M., Barbaro, G., 1996, A\&A, 314, 379

Poggianti, B. M., Smail, I., Dressler, A., Couch, W. J., Barger, A., Butcher, H., Ellis, R.S., Oemler, A., 1999, ApJ, 518, 576

Poggianti, B. M., Bridges, T. J., Mobasher, T. J., Carter, D., et al., 2001a, ApJ, 562, 689 (Paper III)

Poggianti, B. M., Bridges, T. J., Carter, D., Mobasher, T. J., et al., 2001b, ApJ, 563, 118

Postman, M., Lubin, L. M., Oke, J. B. 1998, AJ, 116, 560

Postman, M., Lubin, L. M., Oke, J. B. 2001, AJ, 122, 1125

Rose, J.A., Gaba, A.E., Caldwell, N., Chaboyer, B., 2001, AJ, 121, 793

Skillman, E.D., Tolstoy, E., Cole, A.A., Dolphin, A.E., Saha, A., Gallagher, J.S., Dohm-Palmer, R.C., Mateo, M., 2003, ApJ, in press (astro-ph 0306457)

Smail, I., Ellis, R. S., Dressler, A., Couch, W. J., Oemler, A., Sharples, R.S., Butcher, H., 1997a, ApJ, 479, 70

Smail, I., Dressler, A., Couch, W. J., Ellis, R. S., Oemler, A. Jr, Butcher, H., Sharples, R. M., 1997b, ApJS, 110, 213

Smail, I., Edge, A.C., Ellis, R. S., Blandford, R.D., 1998, MNRAS, 293,124

Stanford, S. A., Eisenhardt, P.R., Dickinson, M., 1998, ApJ, 492, 461

Teague, P.F., Carter, D., Gray, P.M., 1990, ApJS, 72, 715

Tran, K., Franx, M., Illingworth, G.D., van Dokkum, P., Kelson, D.D., 2003, in Carnegie Observatories Astrophysics Series, Vol.3: Clusters of Galaxies: probes of Cosmological Structure and Galaxy Evolution, eds. J.S. Mulchaey, A. Dressler, A. Oemler (Pasadena; Carnegie Observatories: http://www.ociw.edu/ociw/symposia/series/symposium3/proceed

van Dokkum, P. G., Franx, M., Fabricant, D., Illingworth, G.D., Kelson, D. D. 2000, ApJ, 541, 95

Wilson, G., Smail, I., Ellis, R.S., Couch, W.J., 1997, MNRAS, 284, 915

Wirth, G.D., Koo, D.C., Kron, R.G., 1994, ApJ, 435, L105

Zabludoff, A. I., Zaritsky, D., Lin, H., Tucker, D., Hashimoto, Y., Shectman, S.A., Oemler, A., Kirshner, R.P., 1996, ApJ, 466, 104

Ziegler, B., Bender, R., 1997, MNRAS, 291, 527 
TABLE 1

LIST OF K+A GALAXIES.

\begin{tabular}{|c|c|c|c|c|c|c|c|c|}
\hline$\overline{\mathrm{ID}}$ & 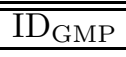 & $\overline{\overline{\mathrm{RA}}(\mathrm{J} 2000)}$ & $\overline{\mathrm{DEC}(\mathrm{J} 2000)}$ & $\overline{\mathrm{R}}$ & $\overline{\overline{(B-R)_{\text {total }}}}$ & vel. & type & $\mathrm{EW}(\mathrm{H} \delta)$ \\
\hline \multicolumn{9}{|c|}{ Blue $\mathrm{k}+\mathrm{a}$ galaxies in Coma1 } \\
\hline 45473 & 3911 & 125855.4 & 275314.58 & 18.79 & 1.25 & 7955 & $\mathrm{k}+\mathrm{a}$ & $9.9 \pm 1.6$ \\
\hline 28211 & 3176 & 125946.3 & 274446.13 & 17.26 & 1.09 & 9739 & $\mathrm{k}+\mathrm{a}$ & $9.4 \pm 0.5$ \\
\hline 30153 & 4060 & 125842.5 & 274537.85 & 16.46 & 0.99 & 8711 & $\mathrm{k}+\mathrm{a}$ & $9.1 \pm 0.6$ \\
\hline 90411 & 3640 & 125915.1 & 281502.96 & 16.60 & 1.00 & 7343 & $\mathrm{k}+\mathrm{a}$ & $8.5 \pm 3.5$ \\
\hline 31086 & 2923 & 130008.2 & 274623.42 & 16.57 & 1.04 & 8664 & $\mathrm{k}+\mathrm{a}$ & $7.4 \pm 0.4$ \\
\hline 6750 & 2498 & 130043.9 & 273536.72 & 17.57 & 1.27 & 7244 & $\mathrm{k}+\mathrm{a}:$ & $6.3 \pm 3.8$ \\
\hline 29362 & 3071 & 125956.2 & 274447.64 & 16.22 & 1.14 & 8931 & $\mathrm{k}+\mathrm{a}$ & $6.0 \pm 0.9$ \\
\hline 90085 & 2989 & 130002.9 & 281424.94 & 16.05 & 1.23 & 7670 & $\mathrm{k}+\mathrm{a}$ & $3.3 \pm 0.3$ \\
\hline 58548 & 3146 & 125948.5 & 275857.80 & 17.67 & 1.32 & 5312 & $\mathrm{k}+\mathrm{a}$ & $3.3 \pm 0.7$ \\
\hline \multicolumn{9}{|c|}{ Red $\mathrm{k}+\mathrm{a}$ galaxies in Coma1 } \\
\hline 63244 & 4003 & 125848.2 & 280108.4 & 17.03 & 1.64 & 7074 & $\mathrm{k}+\mathrm{a}:$ & $5.3 \pm 2.0$ \\
\hline 31300 & 3750 & 125906.7 & 274619.2 & 16.44 & 1.51 & 6292 & $\mathrm{k}+\mathrm{a}:$ & $3.8 \pm 1.1$ \\
\hline 67536 & 2511 & 130043.0 & 280314.4 & 18.21 & 1.69 & 6783 & $\mathrm{k}+\mathrm{a}$ & $3.7 \pm 0.4$ \\
\hline 33963 & 3092 & 125955.0 & 274745.6 & 16.04 & 1.57 & 8016 & $k+a::$ & $3.3 \pm 5.0$ \\
\hline 47098 & 2736 & 130021.6 & 275352.8 & 16.74 & 1.49 & 4856 & $\mathrm{k}+\mathrm{a}$ & $3.2 \pm 0.3$ \\
\hline 52689 & 2692 & 130024.7 & 275537.2 & 16.78 & 1.62 & 7966 & $\mathrm{k}+\mathrm{a}:$ & $3.1 \pm 0.8$ \\
\hline 78794 & 3129 & 125950.2 & 280838.4 & 16.58 & 1.54 & 6849 & $\mathrm{k}+\mathrm{a}::$ & $\perp^{\mathrm{d}}$ \\
\hline \multicolumn{9}{|c|}{$\mathrm{k}+\mathrm{a}$ galaxies in Coma3 } \\
\hline 48397 & 4183 & 125834.1 & 265400.0 & 18.17 & 1.37 & 8114 & $\mathrm{k}+\mathrm{a}$ & $6.9 \pm 2.9$ \\
\hline 149036 & 4215 & 125831.7 & 272342.0 & 17.48 & 1.29 & 7737 & $\mathrm{k}+\mathrm{a}^{\mathrm{b}}$ & $6.5 \pm 1.3$ \\
\hline 169748 & 4597 & 125754.5 & 272927.6 & 15.02 & 1.36 & 4932 & $\mathrm{k}+\mathrm{a}: \mathrm{b}^{\mathrm{b}}$ & $4.8 \pm 2.5$ \\
\hline 76219 & 5065 & 125709.1 & 270148.0 & 18.87 & 1.12 & 7024 & $\mathrm{k}+\mathrm{a}:$ & $4.2 \pm 1.2$ \\
\hline 18086 & 4453 & 125810.1 & 264637.2 & 17.77 & 1.47 & 6748 & $\mathrm{k}+\mathrm{a}$ & $3.7 \pm 0.4$ \\
\hline 154595 & 5250 & 125647.8 & 272515.6 & 15.64 & 1.40 & 7703 & $\mathrm{k}+\mathrm{a}:$ & $3.0 \pm 1.0$ \\
\hline 101924 & 5068 & 125708.6 & 271022.8 & 17.81 & 1.45 & 6005 & $\mathrm{k}+\mathrm{a}::$ & $\mathrm{C}^{\mathrm{d}}$ \\
\hline
\end{tabular}

${ }^{a}$ Possible very weak $\mathrm{H} \alpha$ emission at the $1 \AA$ level.

${ }^{b}$ Other 7 weakest cases (\#55373, 176486, 66948, 95392, 38741, 57916, 81862 in Mobasher et al. (2001)) are not listed here.

${ }^{d}$ The $\mathrm{H} \delta$ line is unmeasurable, but other Balmer lines suggest it could be a $\mathrm{k}+\mathrm{a}$.

TABLE 2

Numbers of SPECTRA OF COMA Galaxies

\begin{tabular}{lccccc}
\hline \hline Cluster & $\mathrm{N}_{\text {tot }}$ & $\mathrm{k}$ & $\mathrm{k}+\mathrm{a} / \mathrm{a}+\mathrm{k}$ & emiss & $?$ \\
\hline Dwarfs 1 & 97 & $26(68)$ & $8(13)$ & $2(4)$ & $12(12)$ \\
Dwarfs 3 & 50 & $19(34)$ & $2(5)$ & $5(6)$ & $5(5)$ \\
Total dwarfs & 147 & $45(102)$ & $10(18)$ & $7(10)$ & $17(17)$ \\
\hline Giants 1 & 80 & $63(70)$ & $2(3)$ & $4(7)$ & $0(0)$ \\
Giants 3 & 33 & $19(20)$ & $1(2)$ & $4(11)$ & $0(0)$ \\
Total giants & 113 & $82(90)$ & $3(5)$ & $8(18)$ & $17(17)$ \\
\hline \multicolumn{7}{l}{ Total "1)" and "3" indicate the numbers in the Coma1 and Coma3 regions. } \\
\hline \multicolumn{7}{c}{ Only galaxies with $R<19$ are included. } \\
\multicolumn{7}{c}{ ? spectra have a poor quality and no spectral class could be assigned. }
\end{tabular}

Numbers without/within brackets exclude/include the uncertain (:) spectra of each class. 
TABLE 3

Fractions of galaxies as a Function of the SPECTral type

\begin{tabular}{lccccc}
\hline \hline Cluster & $\mathrm{N}_{\text {tot }}$ & $\mathrm{k}$ & $\mathrm{k}+\mathrm{a} / \mathrm{a}+\mathrm{k}$ & emiss & $(?)$ \\
\hline Dwarfs 1 & 85 & $0.80 \pm 0.10$ & $0.15 \pm 0.04$ & $0.05 \pm 0.02$ & 0.12 \\
Dwarfs 3 & 45 & $0.76 \pm 0.13$ & $0.11 \pm 0.05$ & $0.13 \pm 0.05$ & 0.10 \\
Total dwarfs & 130 & $0.78 \pm 0.08$ & $0.14 \pm 0.03$ & $0.08 \pm 0.02$ & 0.12 \\
\hline Giants 1 & 80 & $0.88 \pm 0.10$ & $0.04 \pm 0.02$ & $0.09 \pm 0.03$ & 0.00 \\
Giants 3 & 33 & $0.61 \pm 0.14$ & $0.06 \pm 0.04$ & $0.33 \pm 0.10$ & 0.00 \\
Total giants & 113 & $0.80 \pm 0.08$ & $0.04 \pm 0.02$ & $0.16 \pm 0.04$ & 0.00 \\
\hline Total & 243 & $0.79 \pm 0.06$ & $0.09 \pm 0.02$ & $0.12 \pm 0.02$ & 0.07
\end{tabular}

"1" and "3" indicate the numbers in the Coma1 and Coma3 regions.

Fractions were calculated excluding? and including : spectra.

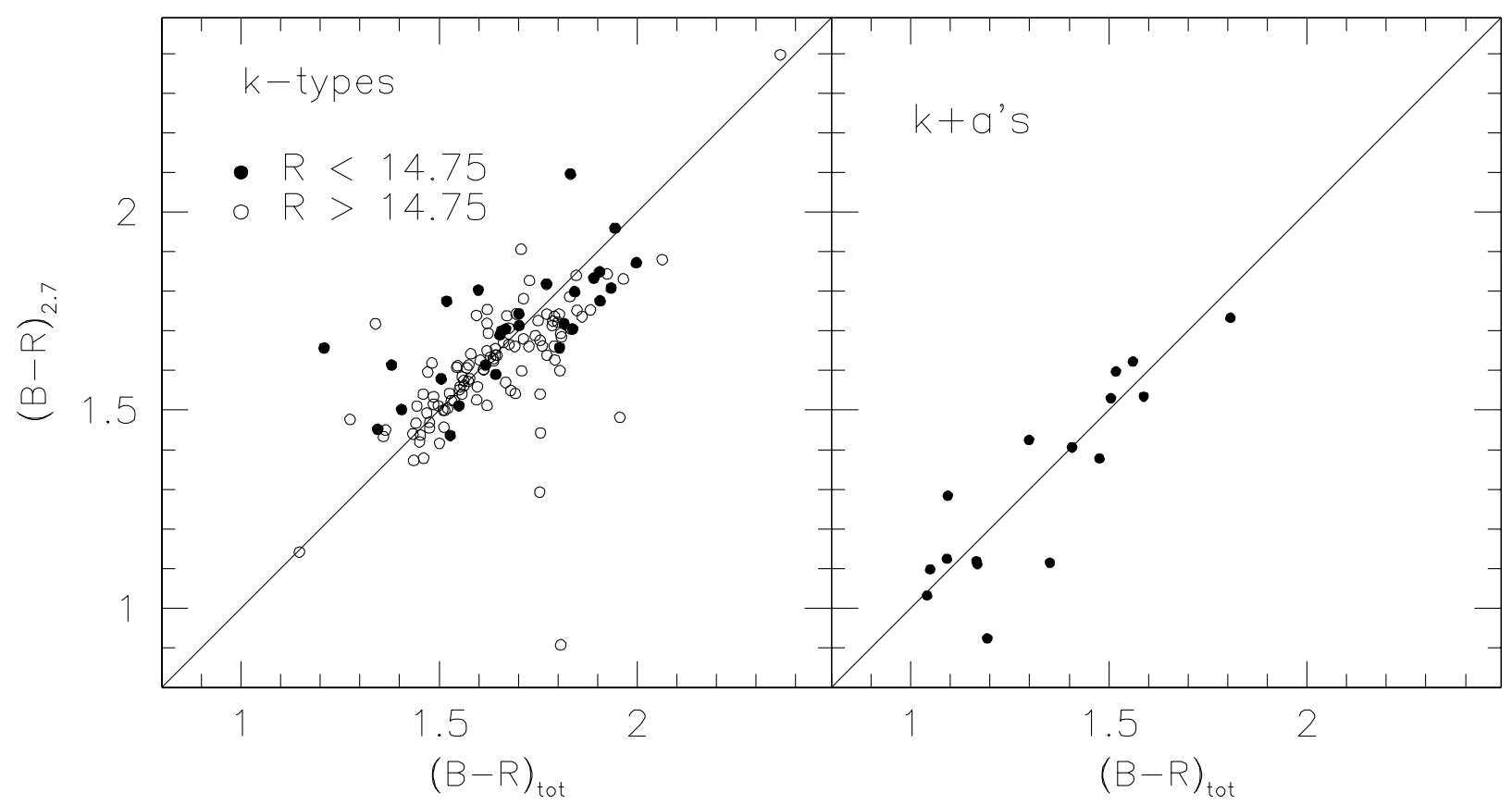

FIG. 1.- Central (2.7" diameter aperture) versus total colors of k galaxies (left) and k+a's (right). Only Coma1 galaxies are presented because Coma3 photometry suffered from saturation in the central pixels of the brightest galaxies. For clarity, errorbars on the colors are not shown. These are typically 0.05 mag. 


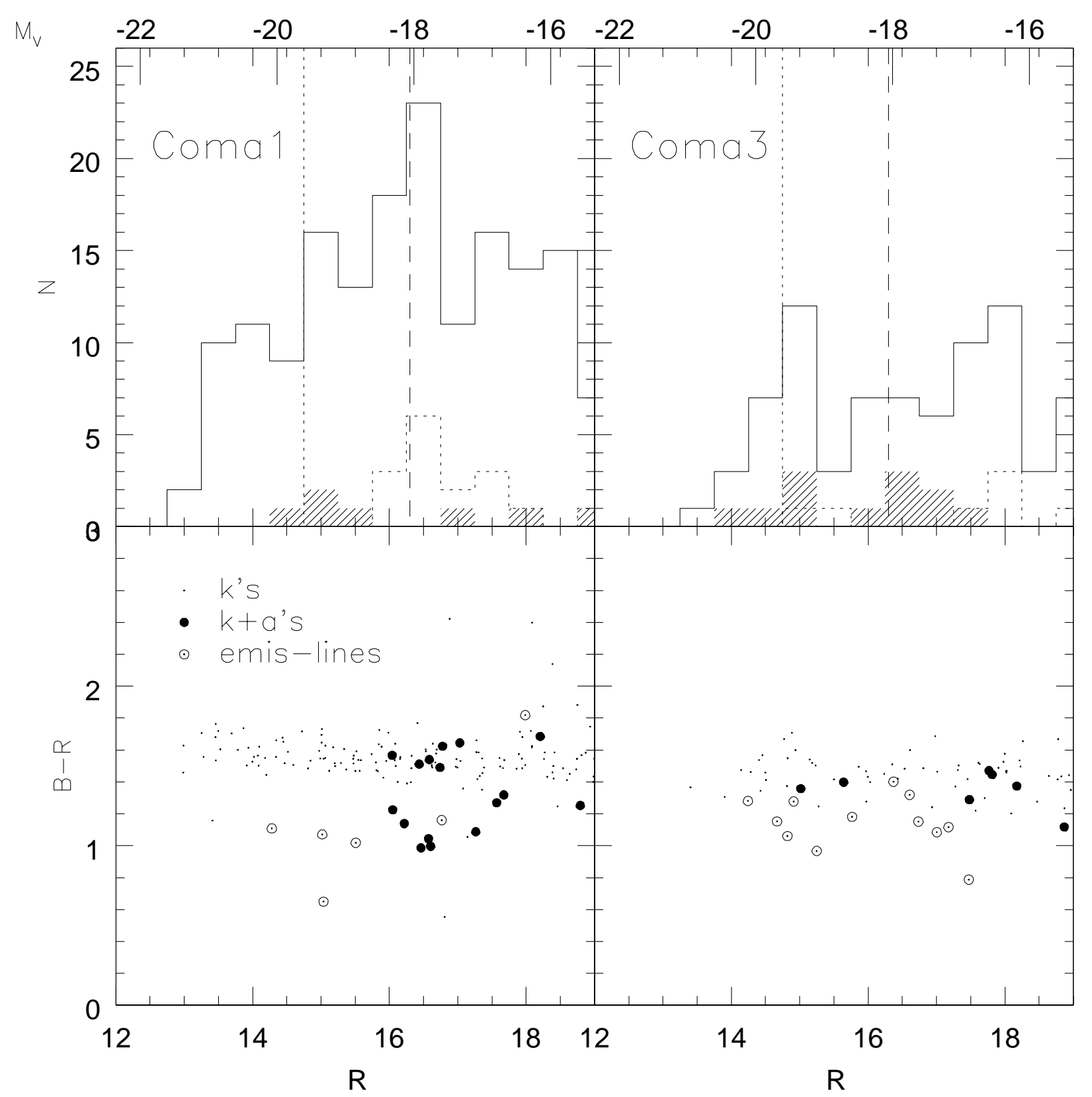

FIG. 2.- Magnitude distributions (top) and color-magnitude diagrams (bottom) of passive, $\mathrm{k}+\mathrm{a}$ and emission-line galaxies with a securely detected line $(\mathrm{EW}([\mathrm{OII}]>2 \AA)$. The dotted line shows the magnitude limit corresponding to the MORPHS cut. The dashed line is our adopted magnitude division between "giants" and "dwarfs". Total histogram= all galaxies with an assigned spectral type. Dashed histogram $=\mathrm{k}+\mathrm{a}$ 's. Shaded histogram $=$ emission-line galaxies. 

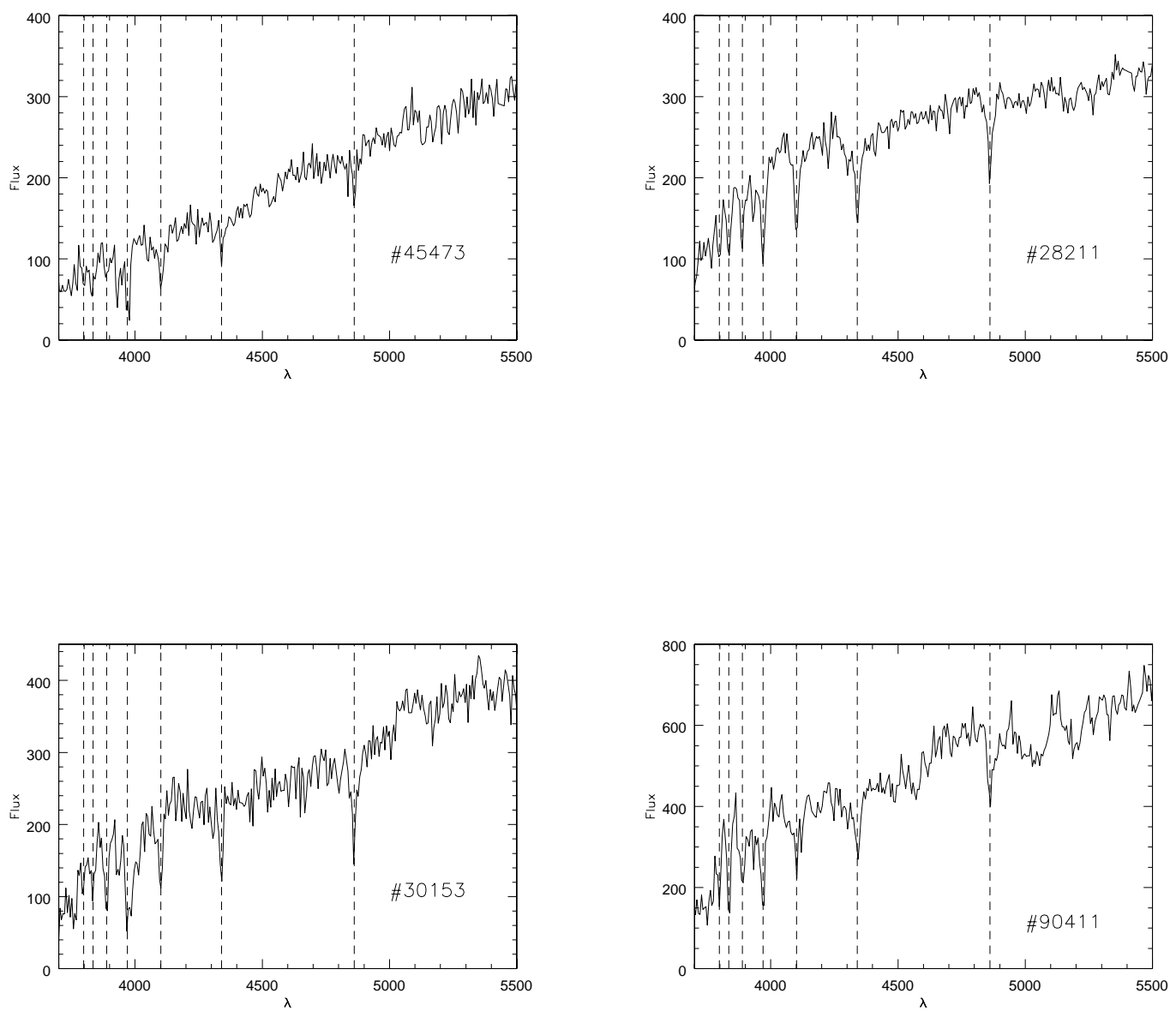

Fig. 3.- Rest-frame spectra of $\mathrm{k}+\mathrm{a}$ galaxies in Coma rebinned at $6 \AA$. The Balmer lines (from left $\mathrm{H} \theta, \mathrm{H} \eta, \mathrm{H} \zeta, \mathrm{H} \epsilon, \mathrm{H} \delta, \mathrm{H} \gamma, \mathrm{H} \beta$ ) are highlighted by dashed lines. In some cases the spectrum includes $\mathrm{H} \alpha$, but the section redder than $5500 \AA$ is not displayed. 

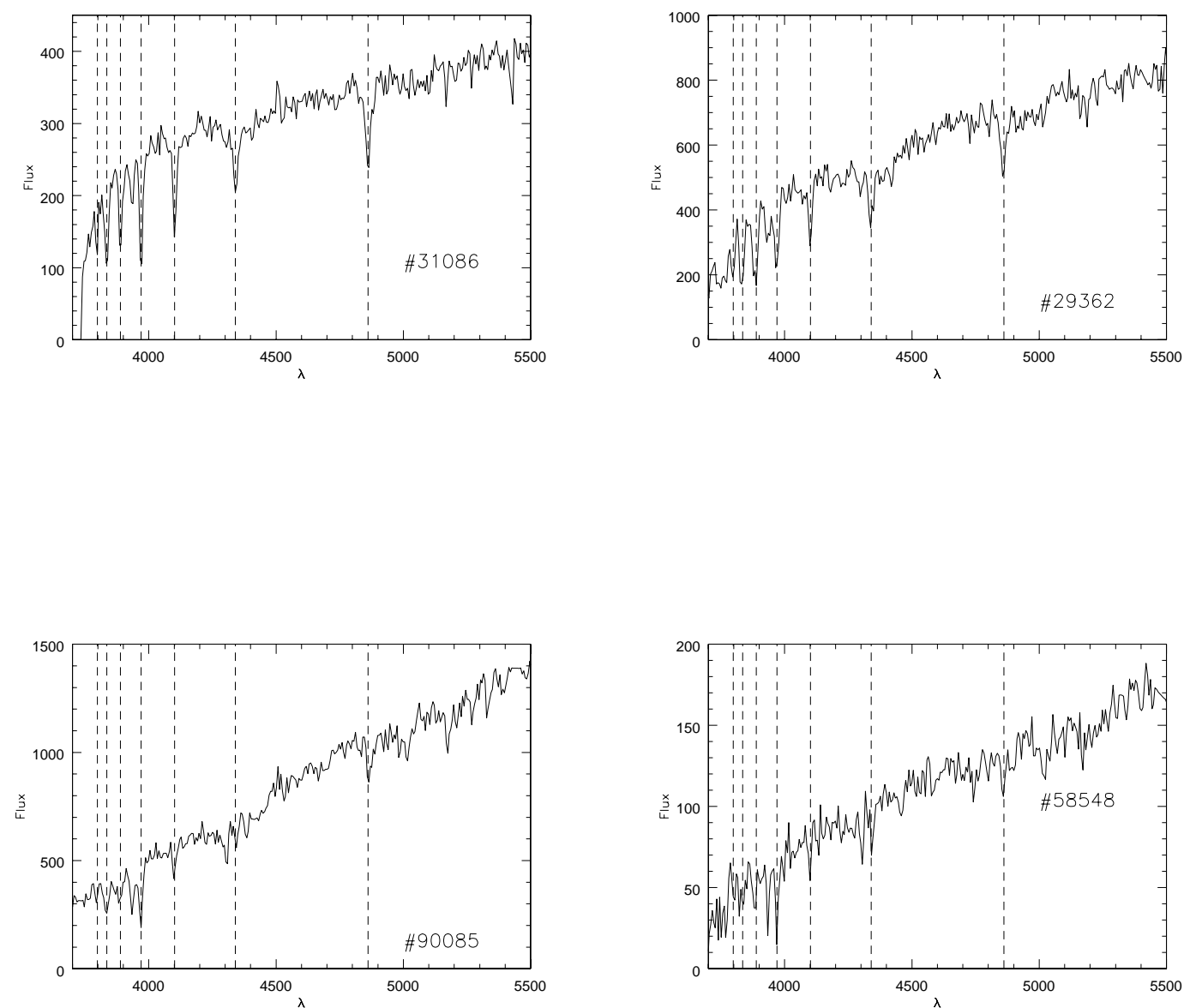

Fig.3 - Continued. 

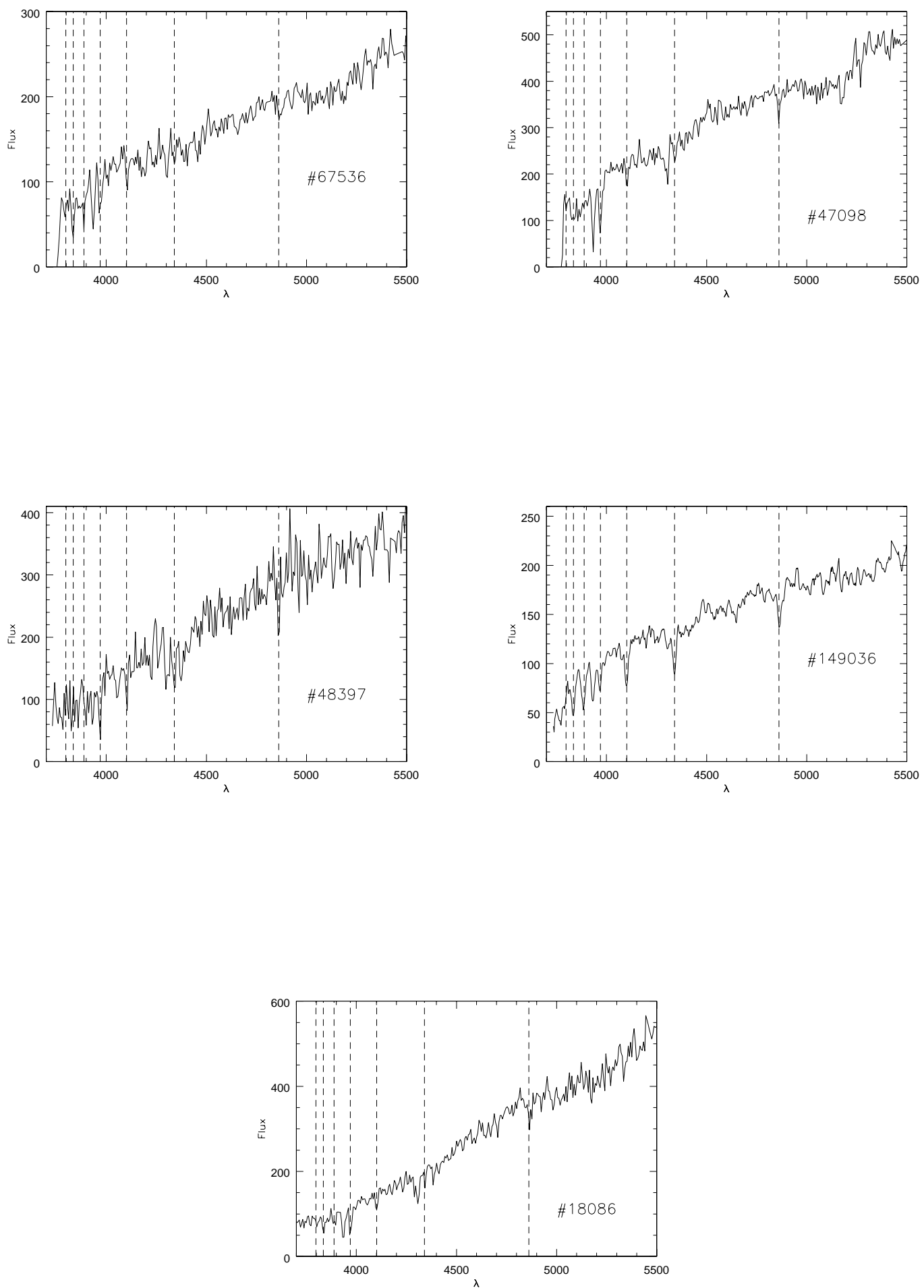

Fig.3 - Continued. 


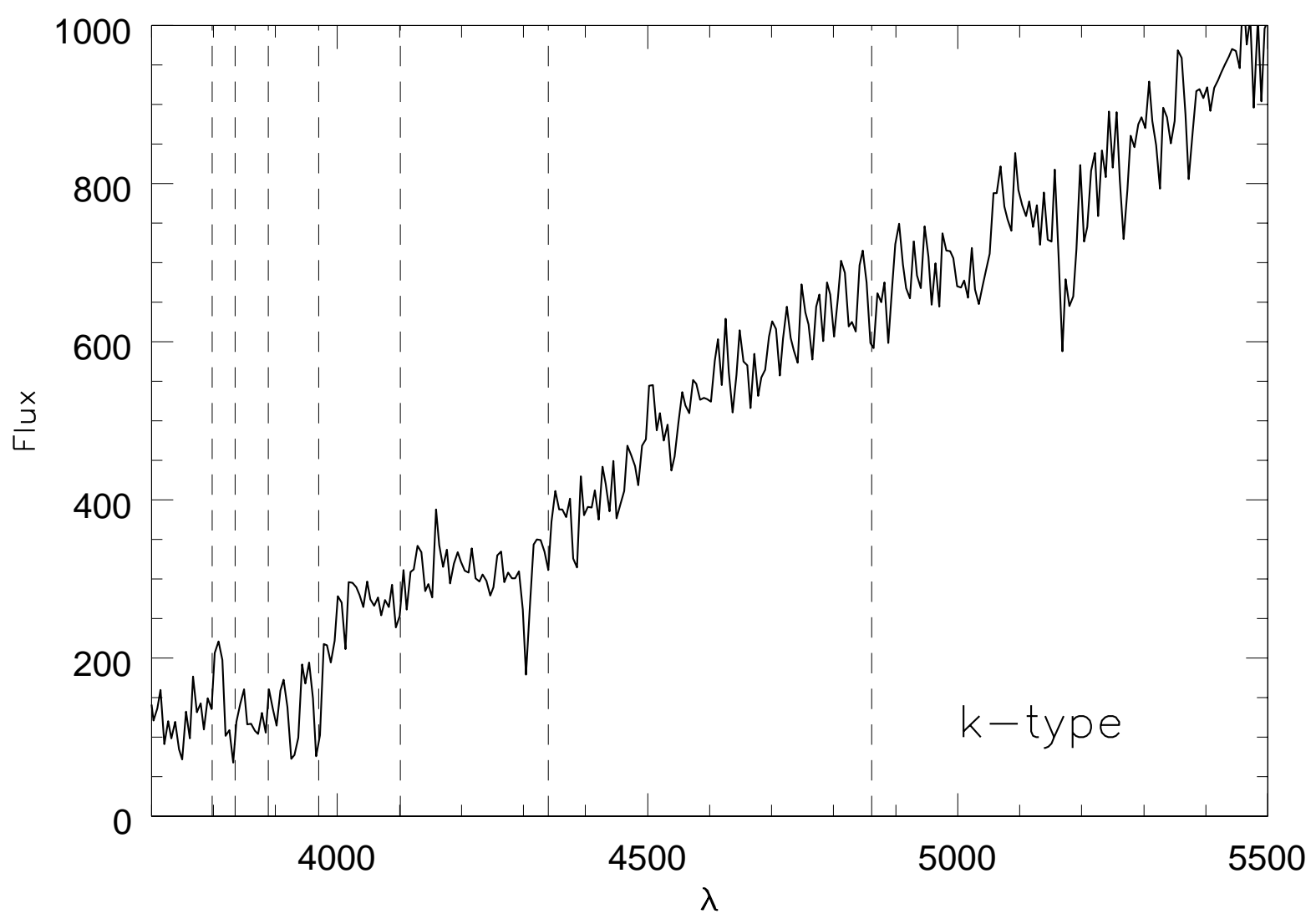

FIG. 4.- Spectrum of a k-type galaxy with similar $\mathrm{R}$ magnitude and $\mathrm{S} / \mathrm{N}$ to the $\mathrm{k}+\mathrm{a}$ spectra shown in the previous figure. 


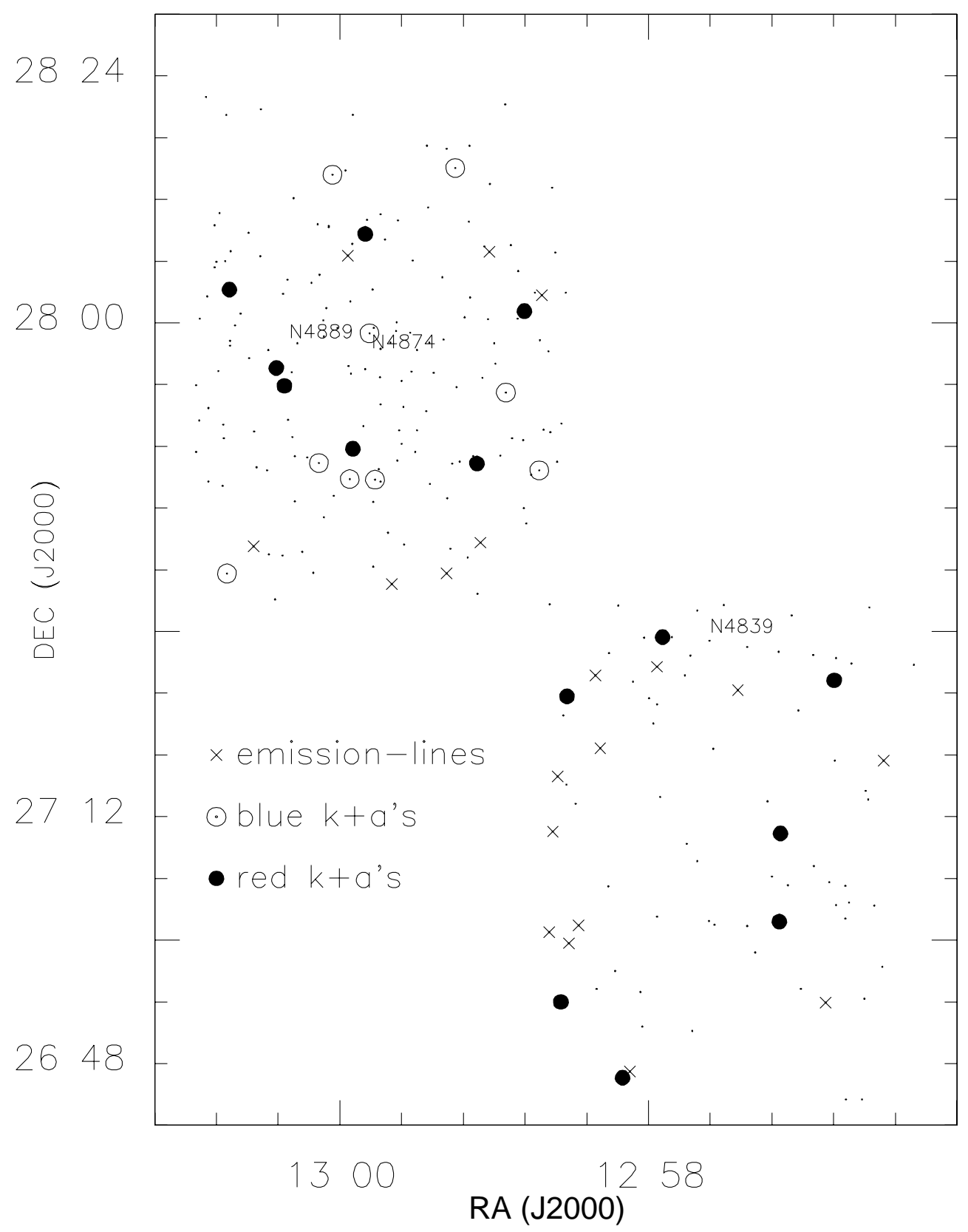

Fig. 5.- Projected position on the sky of $\mathrm{k}$ (small dots), red and blue $\mathrm{k}+\mathrm{a}$ (large circles) and emission-line (crosses) galaxies. The location of the three dominant galaxies (N4874, N4889 and N4839) is labelled. 


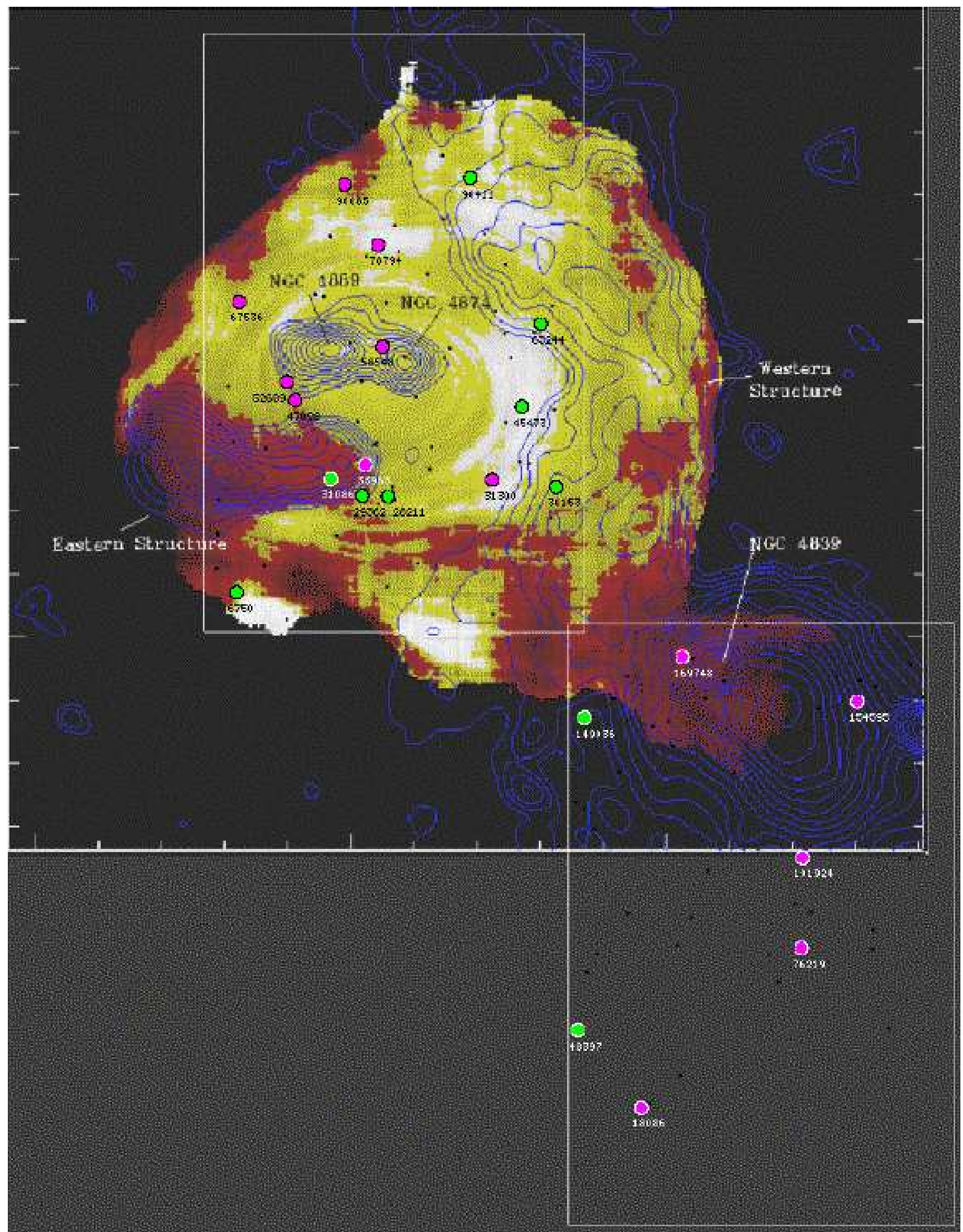

Fig. 6.- Position of $\mathrm{k}+\mathrm{a}$ galaxies with respect to X-ray substructure and X-ray temperature map. Strong-lined k+a's with EW(H $\delta)>5$ $\AA$ are shown as green dots, while weaker $\mathrm{k}+\mathrm{a}$ 's are plotted as magenta dots. Tiny black dots are dwarf Coma members with velocities $7200 \mathrm{km-1}$ - $\mathrm{Y}$, 


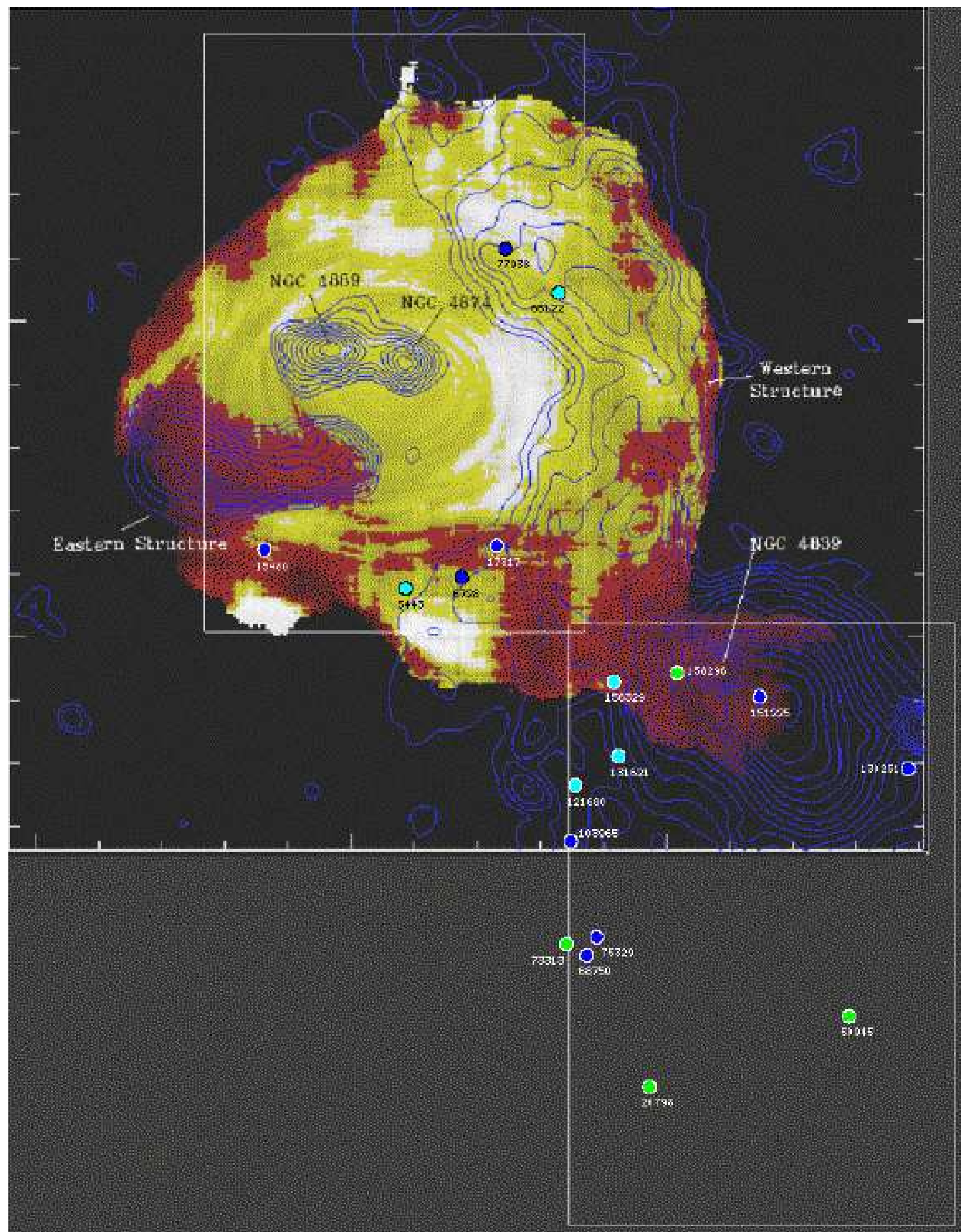

FIG. 7.- Same as Fig. 6, but now showing the positions of emission-line galaxies instead of k+a's. Dark blue dots represent starburst galaxies, green dots are quiescently star-forming galaxies (spiral-like) and light blue dots are emission-line galaxies of unknown activity (either starbursts or spiral-like) 